\title{
Early life vitamin D status and asthma and wheeze: a systematic review and meta-analysis
}

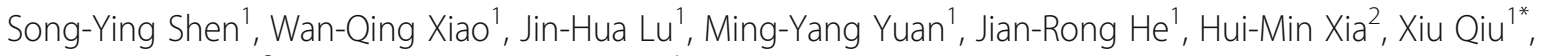
Kar Keung Cheng ${ }^{3}$ and Kin Bong Hubert Lam ${ }^{4}$

\begin{abstract}
Background: Vitamin D deficiency has been linked to an increased risk of asthma. This study aimed to quantify the effect of early life vitamin D status on asthma and wheeze later in life.

Methods: PubMed, Embase, CINAHL, and CNKI databases, the Cochrane Central Register of Controlled Trials, and Google Scholar were searched up to July 2017. We included randomized controlled trials (RCTs) and cohort studies with vitamin D level in blood (maternal or cord or infant) or intake (maternal intake during pregnancy or infant intake) and asthma and/or wheeze. Two reviewers independently extracted data. Fixed- and random-effects models were used to summarize the risk estimates of comparisons between highest vs. lowest vitamin D categories.

Results: Of the 1485 studies identified, three RCTs and 33 cohort studies were included. We did not include the RCTs (1619 participants) in the meta-analysis as the comparators and outcome definitions were heterogenous. Three RCTs reported a non-statistically significant effect of vitamin D supplementation during pregnancy on offspring wheeze/asthma at 3 years of age. Pooled estimates of cohort studies suggest no association between antenatal blood vitamin D levels or vitamin D intake and offspring asthma assessed either $>5$ years or $\leq 5$ years. The estimate for blood vitamin D remained unchanged when two studies assessing asthma in adulthood were excluded, but a significant inverse association emerged between vitamin D intake and childhood asthma. We found no association between antenatal vitamin D level and wheeze. On the other hand, vitamin D intake during pregnancy may have a protective effect against wheeze.

Conclusions: The pooled estimates from cohort studies show no association between antenatal blood vitamin D level and asthma/wheeze in later life. Whereas, the pooled estimates from cohort studies suggest that antenatal vitamin D intake may have an effect on childhood asthma $>5$ years or childhood wheeze. The inconsistent results from studies assessing vitamin $D$ either in blood or intake may be explained by previously reported non-linear association between blood vitamin $\mathrm{D}_{3}$ and childhood asthma. Further trials with enough power and longer followup time should be conducted to confirm the results.
\end{abstract}

Keywords: Vitamin D, Asthma, Wheeze, Pregnancy, Fetal blood, Systematic review, Meta-analysis

\footnotetext{
* Correspondence: xiu.qiu@bigcs.org; qxiu0161@163.com

${ }^{1}$ Division of Birth Cohort Study, Guangzhou Women and Children's Medical

Center, Guangzhou Medical University, 9 Junsui Road, Zhujiang Newtown,

Tianhe District, Guangzhou 510623, China

Full list of author information is available at the end of the article
}

(c) The Author(s). 2018 Open Access This article is distributed under the terms of the Creative Commons Attribution 4.0 International License (http://creativecommons.org/licenses/by/4.0/), which permits unrestricted use, distribution, and

reproduction in any medium, provided you give appropriate credit to the original author(s) and the source, provide a link to the Creative Commons license, and indicate if changes were made. The Creative Commons Public Domain Dedication waiver (http://creativecommons.org/publicdomain/zero/1.0/) applies to the data made available in this article, unless otherwise stated. 


\section{Background}

Asthma is an important cause of disability and a major worldwide public health concern. The prevalence of asthma in developed countries rose rapidly in the 1960s and has remained high since [1]. More recently, the same rising trend has been observed in developing countries [2]. While asthma affects people of all ages, the surge among children has been most marked [3].

Among the environmental factors that contribute to asthma, vitamin D status has generated increasing interest for the vitamin's purported immunomodulatory properties.

Clinically, serum calcifediol $[25(\mathrm{OH}) \mathrm{D}]$ is used as a marker of vitamin D level [4]. In various populations calcifediol levels in cord blood are strongly correlated with maternal levels during pregnancy [5], with maternal calcifediol as the source of the fetal vitamin D pool [6]. Vitamin D deficiency in pregnant women and infants is common worldwide, including both developed and developing countries, ranging from 45 to $90 \%$ in pregnant women and $61-94 \%$ in infants [5, 7-9]. Based on growing epidemiological evidence, vitamin D deficiency has been linked to an increased risk of respiratory infections and asthma [10]. However, it is still unclear if and to what extent antenatal or early postnatal vitamin $\mathrm{D}$ deficiency would affect the development of wheeze or asthma later in life. Previous reviews on vitamin D supplementation during pregnancy have given conflicting messages [11-13].

Given that there have been further published studies (including trials), we undertook a systematic review aiming to address whether antenatal or early postnatal vitamin D status (including intake and blood level, both maternal and in infant) has any impact on the risk of developing asthma and wheeze later in life.

\section{Methods}

We followed the Meta-analysis of Observational Studies in Epidemiology (MOOSE) [14] and the Preferred Reporting Items for Systematic Reviews and Meta-Analyses (PRISMA) guidelines [15] when conducting and presenting this systematic review and meta-analysis. PRISMA and MOOSE checklist can be found in Additional file 1: Supplement 1. The review protocol was registered previously with the International Prospective Register of Systematic Reviews (PROSPERO Registration No. CRD42013005559) [16]. The initial protocol considered asthma/wheeze, allergic rhinitis, atopic dermatitis (eczema), food allergy and atopic sensitization, but here we focus on asthma and wheeze.

\section{Data sources and searches}

We conducted a systematic literature search using PubMed, Embase, CINAHL, and CNKI (in Chinese) databases and Google Scholar for studies published up to 19th July 2017 and the Cochrane Central Register of Controlled Trials that reported data on antenatal or early postnatal vitamin D status or intervention and asthma and wheezing in children or adults. Details of the search strategy are provided in Additional file 1: Supplement 2.

\section{Study selection}

The present systematic review focused on the effect of vitamin D level in blood (maternal or cord or infant) and intake (maternal intake during pregnancy or infant intake) on asthma and wheeze. We included randomized controlled trials (RCTs), quasi-randomized controlled trials, non-randomized controlled trials, prospective and retrospective cohort studies that measured maternal vitamin D status during any trimester of pregnancy or cord blood or offspring vitamin D status during infancy, and having asthma or wheeze during childhood or adulthood as outcomes, which (i) were diagnosed by doctors (including parental or self-reports), or (ii) required the use of asthma medication, or (iii) as assessed by the International Study of Asthma and Allergies in Children (ISAAC) questionnaire (Additional file 1: Supplement 3). Two reviewers (SS and $\mathrm{WX}$ ) independently screened the titles and abstracts and identified potentially relevant publications according to the selection criteria, of which full text was obtained and read (online Additional file 1: Supplement 4). The reference lists of all papers of interest were scrutinized to obtain other relevant articles. Disagreements over inclusion were resolved through consensus, and where necessary, a third reviewer (JL) was involved.

\section{Data extraction and risk of Bias assessment}

The same reviewers independently extracted data using a standard data extraction form. We collected data on the number of subjects with and without asthma or wheeze in the antenatal or early postnatal vitamin D exposed/intervention and non-exposed/control groups; risk estimates (crude and/or adjusted odds ratios [ORs], relative risks [RRs] and hazard ratios [HRs]) and the corresponding 95\% confidence intervals (CIs) at any available age end point. Furthermore, we recorded information on the population, geographical location, inclusion and exclusion criteria, interval of follow up, exposure measurement, outcome definition, measurement and window of assessment, and confounders (more details in Additional file 1: Supplement 5). Study authors were contacted when missing data was an issue. Disagreements on data extraction were checked against the original articles and/or resolved by a third reviewer. The data extracted were entered into Review Manager software and were double checked by two reviewers. Risk of bias was assessed using the tool recommended by the Cochrane Collaboration $[17,18]$. Two reviewers independently rated the risk of bias for each study as high, low, or unclear ('Definitely yes' in the tool was considered as 'low' risk, 'Definitely no' as 'high' risk, 'unclear' 
comprised 'probably yes' and 'probably no' as specified in the tools [18]). Disagreements were resolved by discussion or by involving a third reviewer. Each cohort study was classified into one of the following categories: high (more than two criteria not applied/met); moderate (one or two criteria not applied/met or unclear); and low risk of bias (all criteria applied/met).

\section{Data synthesis and analysis}

We pooled the original risk estimates as reported (most being ORs) and used adjusted risk estimates where available. When only frequency distributions were provided, we calculated unadjusted ORs and their 95\% CIs from the outcome distribution of exposed and non-exposed groups. Where outcome assessment was done in multiple time points in a study, we chose the one with the longest follow-up period or the time point when the age of the participants was comparable to other studies.

We pooled the risk estimates from each study that compared the risk in the highest vitamin D concentration or intake category with the lowest (which is the referent category), and also the risk in vitamin $D$ sufficiency $(\geq 75 \mathrm{nmol} / \mathrm{L}[4])$ with deficiency $(<50 \mathrm{nmol} / \mathrm{L} \mathrm{ac}-$ cording to the Institute of Medicine (IOM) definition [19]). As it could be difficult to diagnose asthma in children below 5 years of age, we stratified the studies on asthma according to the age of outcome assessment ( $>5$ years and $\leq 5$ years). For exploring the association between blood vitamin D status and childhood asthma, a sensitivity analysis was performed by excluding the studies assessing the outcome in adulthood. We contacted the authors of the studies $(n=8)$ that had different cut-off values, and asked them to re-analyze their data according to the above categorization scheme. Of the eight authors contacted, four responded and provided additional data [20-23].

Both fixed-effects and random-effects models were used to summarize the effect sizes. Heterogeneity was evaluated by using the $\mathrm{Q}\left(P>0.10\right.$ in the $\mathrm{Chi}^{2}$ test for low heterogeneity) and $\mathrm{I}^{2}$ statistics (moderate heterogeneity for $\mathrm{I}^{2}>30 \%$ and considerable heterogeneity for $\geq 75 \%$ ). We presented pooled ORs from random-effect models when considerable heterogeneity was observed. Stratified analyses by time window of vitamin D and outcome assessment (for wheeze only), latitude, geographical region, adjustment for family history of allergic disease, seasonality, and smoking status, methods of exposure and outcome ascertainment, and risk of bias were performed to explore the sources of heterogeneity. Funnel plots and Egger's test were used to explore the possibility of publication bias. Number needed to treat for preventing asthma by increasing from low to high levels of vitamin D or via supplementation was estimated by the baseline asthma prevalence estimates and pooled ORs [24]. Statistical analyses were performed with Review Manager software version 5.3 [25] and STATA version 13 .

\section{Results}

\section{Search results}

We have identified 423 potentially relevant publications from PubMed, 855 from Embase, 36 from CNKI database, 105 from CINAHL and 66 from Google Scholar. After excluding duplicates and publication that did not meet the inclusion criteria, three RCTs [26-28] and 33 cohort studies [20-23, 29-57] were included (Fig. 1).

\section{Study characteristics}

The main characteristics of the eligible RCTs, cohort studies and those studies excluded during full text screening are shown in Tables 1 and 2 and Additional file 1: Table S1, respectively. Two of the three RCTs were single-center trials and recruited low-risk pregnant women. The other was a multicenter trial enrolling pregnant women who had history of allergic disease. The dose of vitamin D administration and outcome definition varied across the three trials (1619 participants) (Table 1). The median ages of outcome assessment in cohort studies that reported association between blood vitamin D level and asthma, vitamin D intake and asthma, blood vitamin D and wheeze, and vitamin D intake and wheeze were 6 years (range 1.5-25), 5 (1.5-31), $5(1-10)$, and $3(1-10)$, respectively. Only two studies assessed the outcome in adulthood $[36,57]$. Of the cohort studies, 26 studies reported data on asthma and 17 on wheeze. Nineteen of these 26 asthma studies measured vitamin D levels (31,940 participants from 13 cohorts were included in the meta-analysis); the rest assessed dietary/ supplement intake (41,952 participants). A variety of operational definitions of asthma were used, including parental reports of doctor-diagnosed asthma $(n=7)$, parental reports of asthma medication use $(n=1)$, a combination of both $(n=2)$, parental reports of doctor-diagnosed asthma with wheezing symptom $(n=4)$, parental reports of doctor-diagnosed asthma and wheezing and/or asthma medication use $(n=5)$ and doctor-diagnosed asthma or asthma medication use by medical record review $(n=5)$. Two studies did not report the definition of asthma. All 17 studies on wheeze assessed maternal vitamin $\mathrm{D}$ status during pregnancy, with 12 measuring blood vitamin D levels (8123 participants from 8 cohorts were meta-analyzed) and 6 recorded intake (5678 participants). Most wheeze studies defined outcome using parental reports of wheezing symptoms in the past 12 months (Table 2).

\section{Early life vitamin D status and asthma}

Two RCTs $[27,28]$ reported non-significant trends of vitamin D supplementation during pregnancy on preventing 


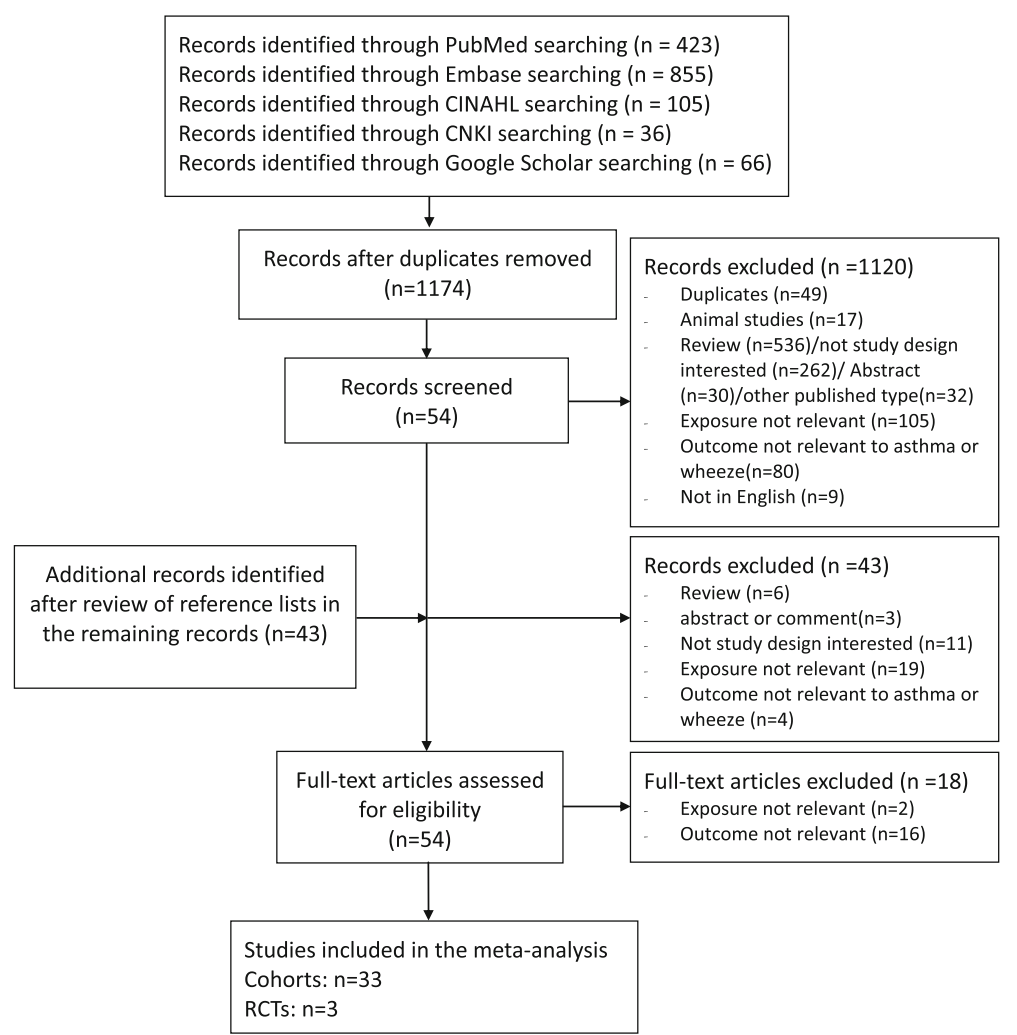

Fig. 1 Summary of literature search and study selection

Table 1 Characteristics of the randomized controlled trials

\begin{tabular}{|c|c|c|c|c|c|}
\hline Reference & Design & Patients characteristics & $\begin{array}{l}\text { Intervention } \\
\text { (Number of participants) }\end{array}$ & $\begin{array}{l}\text { Comparator } \\
\text { (Number of participants) }\end{array}$ & $\begin{array}{l}\text { Outcomes assessment and } \\
\text { definition (Age of outcome } \\
\text { assessment) }\end{array}$ \\
\hline $\begin{array}{l}\text { Goldring, } \\
2013[26]\end{array}$ & $\begin{array}{l}\text { Single-center } \\
\text { non-blinded } \\
\text { non-placebo } \\
\text { RCT }\end{array}$ & $\begin{array}{l}\text { Pregnant women in } \\
\text { London }\left(51^{\circ} \mathrm{N}\right) \text { ( } 27 \text { weeks } \\
\text { gestation, Asian, Middle } \\
\text { Eastern, Black and White) } \\
\text { without sarcoidosis, } \\
\text { osteomalacia, renal } \\
\text { dysfunction or tuberculosis }\end{array}$ & $\begin{array}{l}\text { 1. Daily vitamin D } \\
\text { ( } 800 \text { IU ergocalciferol } \\
\text { until delivery) }(n=60) \\
\text { 2. Bolus vitamin D } \\
\text { (a single oral dose of } \\
200,000 \text { IU } \\
\text { cholecalciferol }(n=60)\end{array}$ & No treatment $(n=60)$ & $\begin{array}{l}\text { 1.Wheeze ever (ISAAC, at age } 3 \text { ) } \\
\text { 2. Recurrent wheezing ( } \geq 2 \\
\text { episodes of reported wheezing } \\
\text { since birth, at age } 3 \text { ) } \\
\text { 3. Wheeze in the year prior to } \\
\text { assessment (ISAAC, at age } 3 \text { ) } \\
\text { 4. Wheeze with a positive } \\
\text { asthma predictive index } \\
\text { (loose criteria, at age } 3 \text { ) }\end{array}$ \\
\hline $\begin{array}{l}\text { Chawes, } \\
2016 \text { [27] }\end{array}$ & $\begin{array}{l}\text { Single-center } \\
\text { double-blinded RCT }\end{array}$ & $\begin{array}{l}\text { Pregnant women in } \\
\text { Denmark }\left(55^{\circ} 43^{\prime} \mathrm{N}\right) \\
\text { ( } \leq 26 \text { gestational week) } \\
\text { without any endocrine, } \\
\text { cardiovascular, or } \\
\text { nephrological disorders; } \\
\text { or vitamin D3 } \\
\text { (cholecalciferol) intake } \\
\text { more than } 600 \mathrm{lU} / \mathrm{d}\end{array}$ & $\begin{array}{l}\text { Daily dose of } 2400 \text { IU } \\
\text { vitamin D3 } \\
\text { supplementation } \\
\text { ( } 24 \text { gestational week to } \\
1 \text { week postpartum) } \\
\text { ( } n=315)\end{array}$ & $\begin{array}{l}\text { Matching placebo } \\
\text { tablets (Camette A/S) } \\
\text { ( } 24 \text { gestational week to } \\
1 \text { week postpartum) } \\
(n=308)\end{array}$ & $\begin{array}{l}\text { 1.Persistent wheeze (diagnosed } \\
\text { according to a previously } \\
\text { validated quantitative algorithm, } \\
\text { from birth to age } 3 \text { years) } \\
\text { 2.Asthma (doctor diagnosed in } \\
\text { children fulfilling the persistent } \\
\text { wheeze criteria at age } 3 \text { ) }\end{array}$ \\
\hline $\begin{array}{l}\text { Litonjua, } \\
2016 \text { [28] }\end{array}$ & $\begin{array}{l}\text { Multicenter } \\
\text { double-blinded } \\
\text { placebo RCT }\end{array}$ & $\begin{array}{l}\text { Pregnant women in } \\
\text { Boston }\left(52^{\circ} 58^{\prime} \mathrm{N}\right) \text {, } \\
\text { Washington }\left(38^{\circ} 53^{\prime} \mathrm{N}\right) \text {, } \\
\text { San Diego }\left(32^{\circ} 43^{\prime} \mathrm{N}\right) \\
\text { (age } 18-39,10-18 \text { gestational } \\
\text { week, nonsmoker, English or } \\
\text { Spanish speaking, who or } \\
\text { whose partner had a } \\
\text { history of allergic disease, } \\
\text { with intent to participate } \\
\text { for } 4 \text { years) }\end{array}$ & $\begin{array}{l}\text { Daily } 4000 \mathrm{IU} \text { of vitamin } \\
\text { D plus a multivitamin } \\
\text { with } 400 \mathrm{IU} \text { of vitamin D } \\
(n=440)\end{array}$ & $\begin{array}{l}\text { Placebo (daily placebo } \\
\text { pill plus a multivitamin } \\
\text { with } 400 \text { IU of vitamin D) } \\
(n=436)\end{array}$ & $\begin{array}{l}\text { Asthma or recurrent } \\
\text { wheeze in first } 3 \text { y of life } \\
\text { (parental report, every } 3 \text { months) }\end{array}$ \\
\hline
\end{tabular}




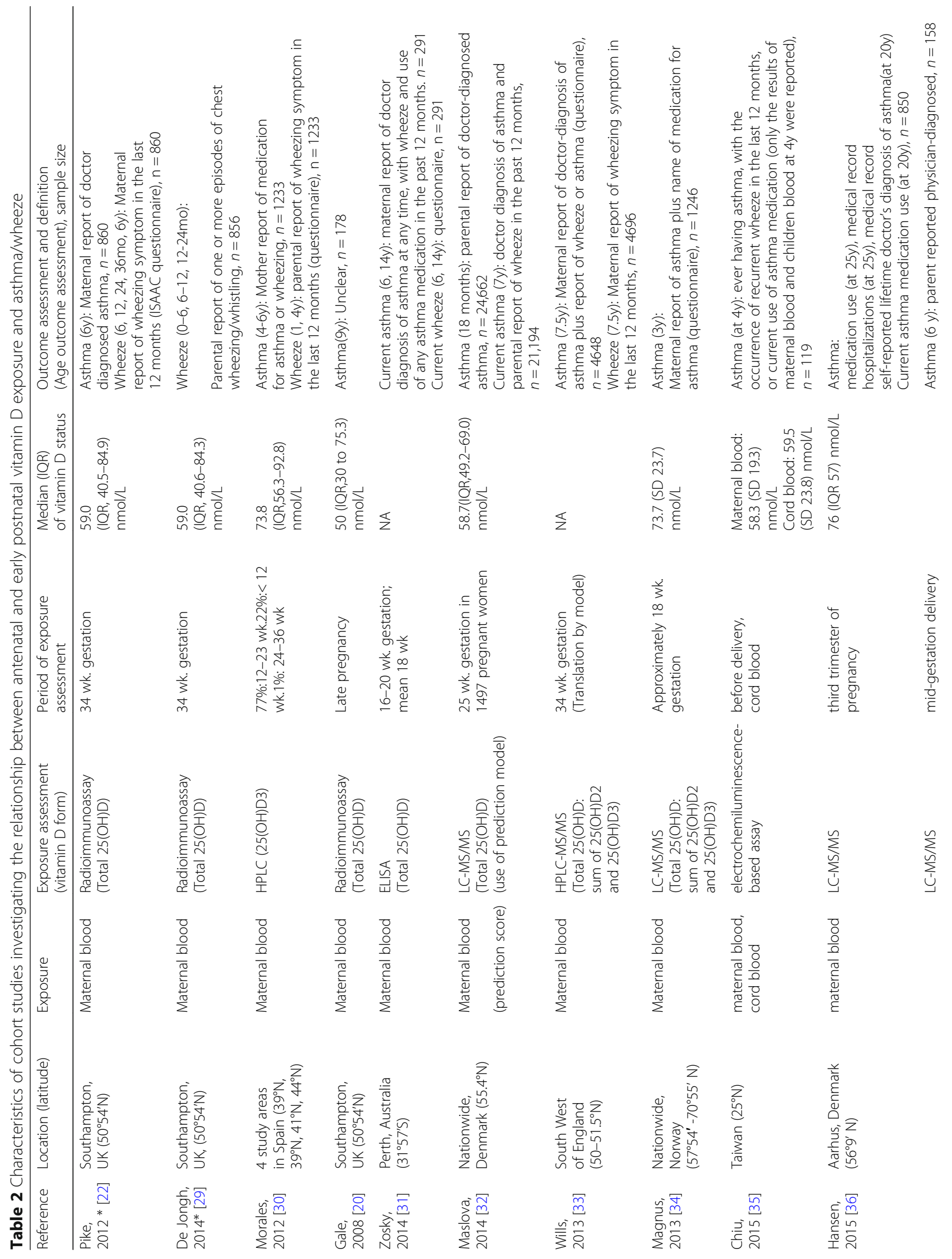




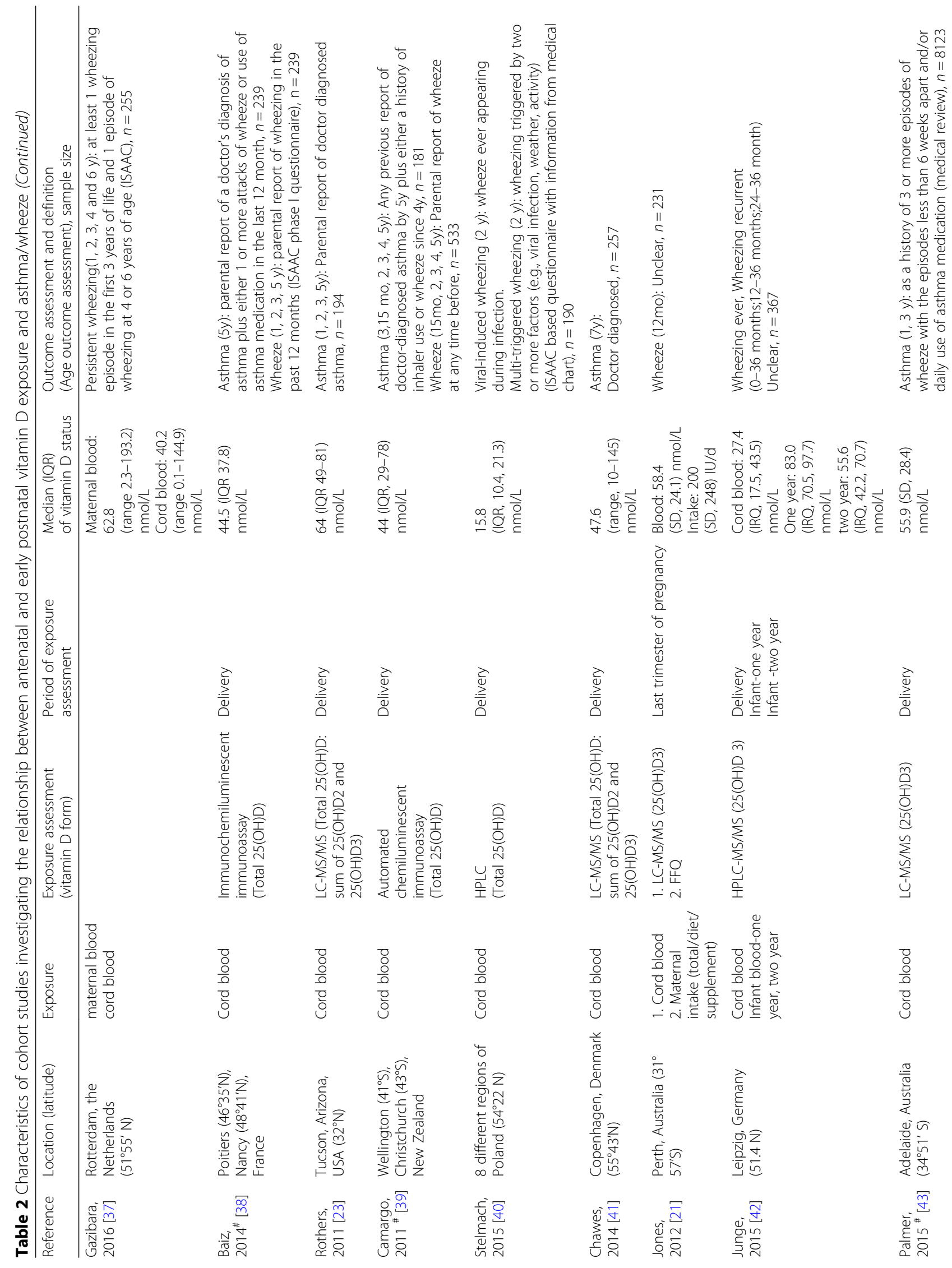




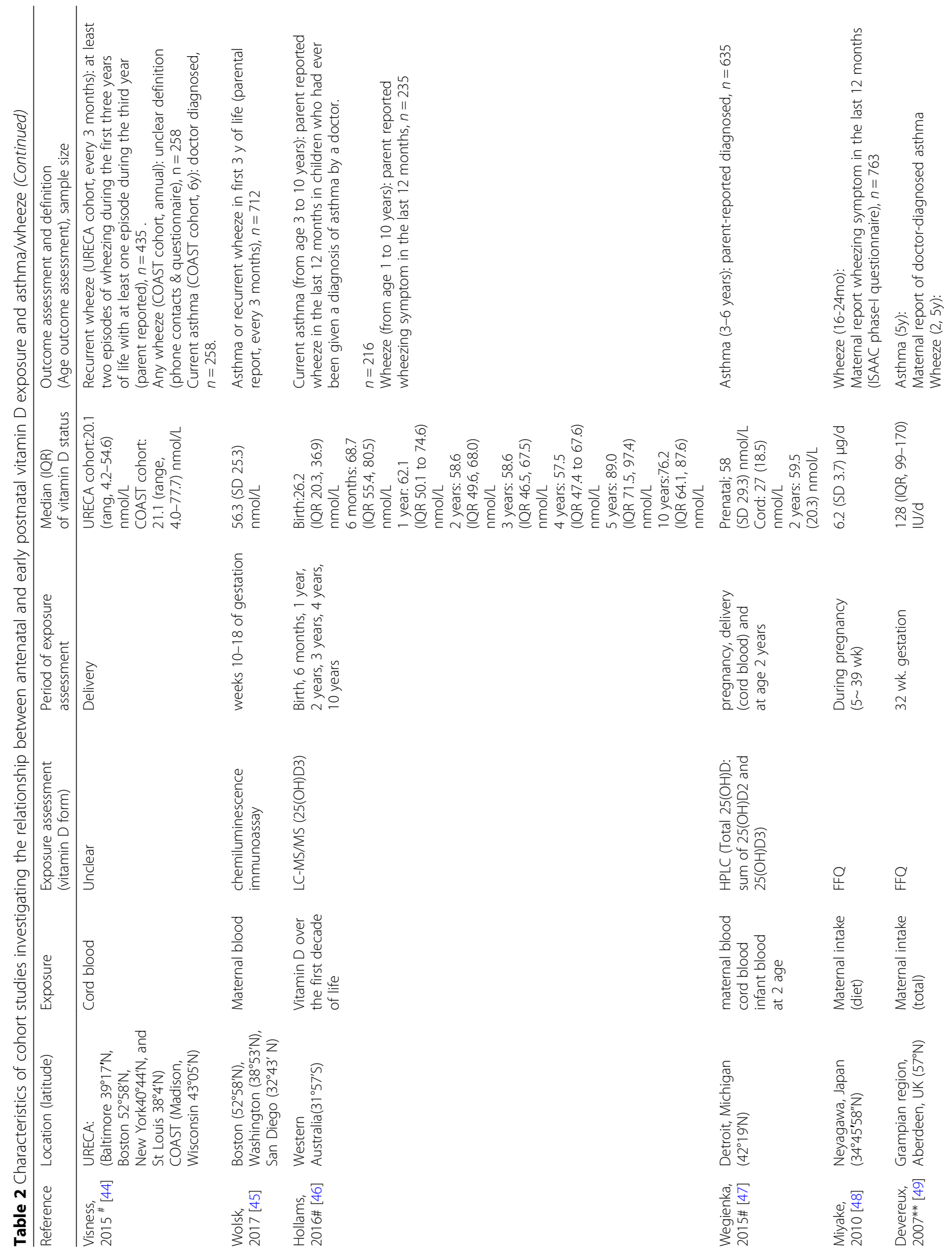




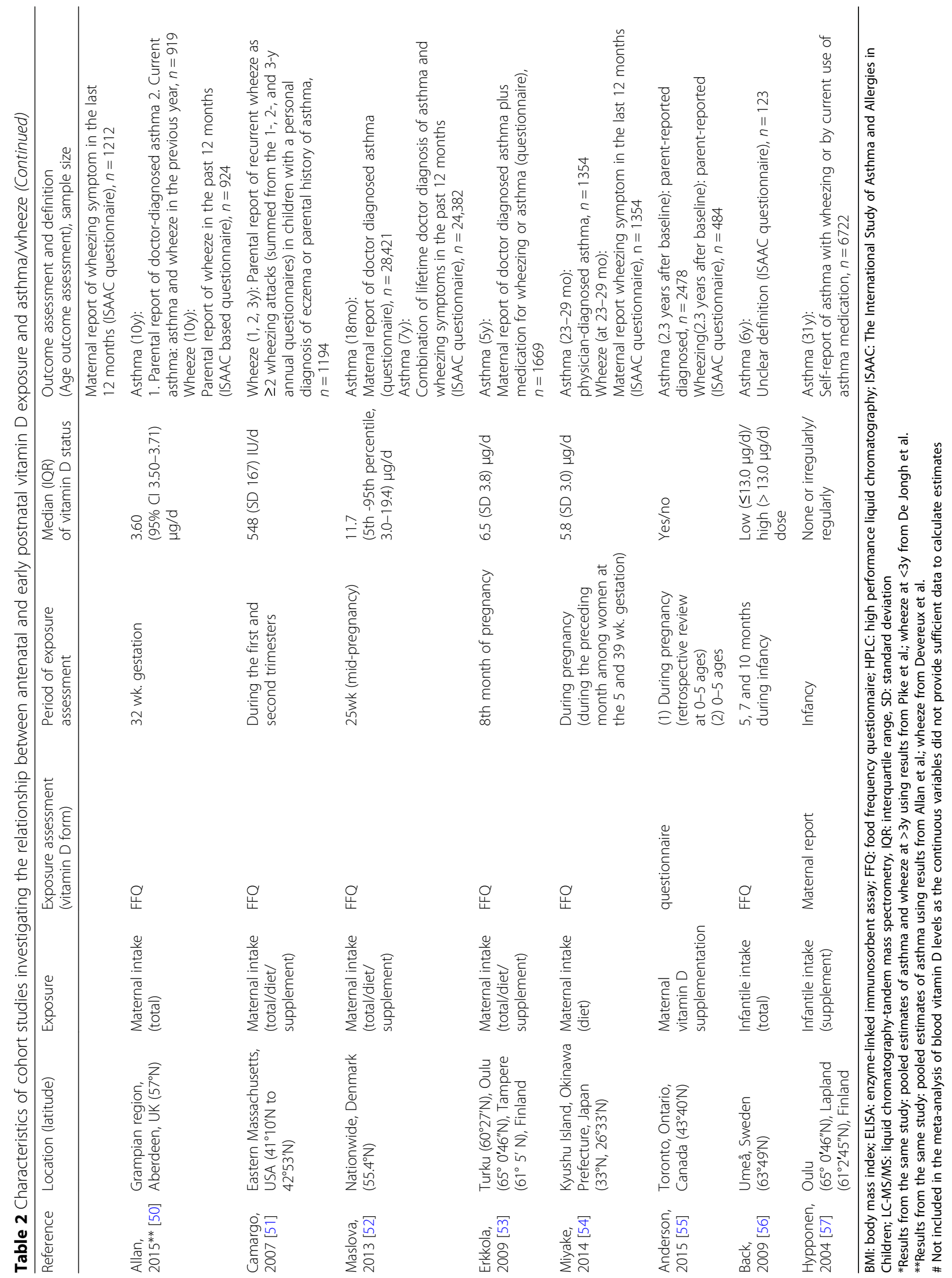


the development of offspring asthma (OR, 0.82; 95\% CI $0.50-1.36$ and $\mathrm{HR}, 0.8$; 95\% CI, 0.6-1.0, respectively). As the intervention and the outcome definition were different, we did not pool the effect size. Apart from having insufficient information to assess allocation concealment, risk of bias was low in both RCTs (Additional file 1: Figure S1).

Figure 2 and Table 3 show the results from cohort studies as well as overall and subgroup summary ORs for the relationship between blood vitamin D and the risk of asthma assessed at $>5$ years of age. Fix-effects ORs were not significant for the highest vs. the lowest categories of blood vitamin D concentrations (8 cohort studies, 28,436 participants, Fig. 2a) and for the sufficient vs. deficient groups ( 5 cohort studies, 23,339 participants, Fig. $2 b$ ), with moderate heterogeneity. The pooled estimate did not change materially (OR $0.91,95 \%$ CI $0.73,1.12, \mathrm{I}^{2}=32 \%$, for highest vs. lowest; OR 1.02, 95\% CI $0.84,1.24, \mathrm{I}^{2}=47 \%$ for sufficient vs. deficient groups) after excluding one study assessing asthma in adulthood. Little heterogeneity was observed across subgroups, in studies measuring cord blood vitamin D level, adjusted for family history of allergic disease, or seasonality, those with exposure measured by liquid chromatography-tandem mass spectrometry (LC-MS/MS), and studies with moderate risk of bias (Table 3 ).

Figure 3 and Table 3 show the results from cohort studies and the overall and subgroup summary ORs for the relationship between blood vitamin $\mathrm{D}$ and the risk of asthma assessed at $\leq 5$ years of age. The fixed-effects ORs were $0.81\left(95 \%\right.$ CI $0.65,1.01, \mathrm{I}^{2}=0 \%, 6$ cohort studies, 27,776 participants, Fig. 3a) for the highest vs. lowest categories of blood vitamin D concentrations and 0.93 for the sufficient vs. deficient groups $\left(95 \%\right.$ CI $0.85,1.03, \mathrm{I}^{2}=$ 36\%, 6 cohort studies, 27,776 participants, Fig. 3b). Although higher maternal blood vitamin $\mathrm{D}$ level during pregnancy was significantly associated with lower risk of asthma assessed at $\leq 5$ years, we did not observe any

\section{a Highest category vs. lowest category of vitamin D}

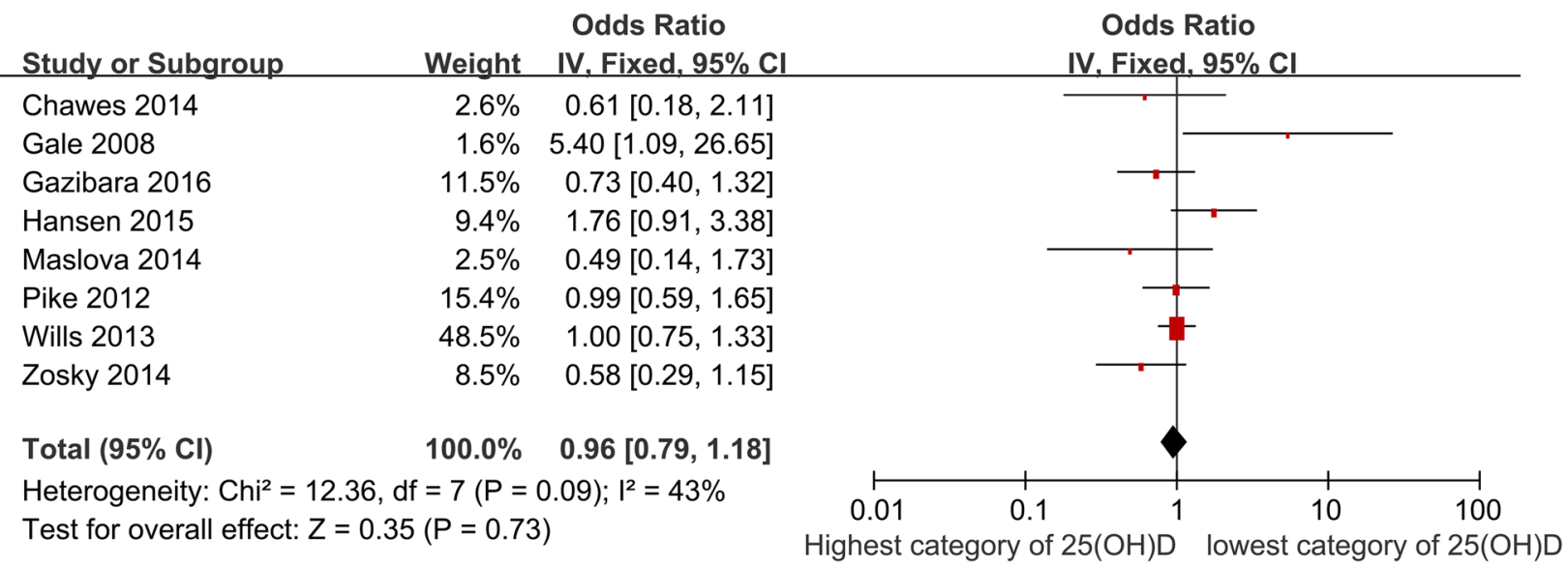

\section{b $\geq 75 \mathrm{nmol} / \mathrm{L}$ vs. $<50 \mathrm{nmol} / \mathrm{L}$}

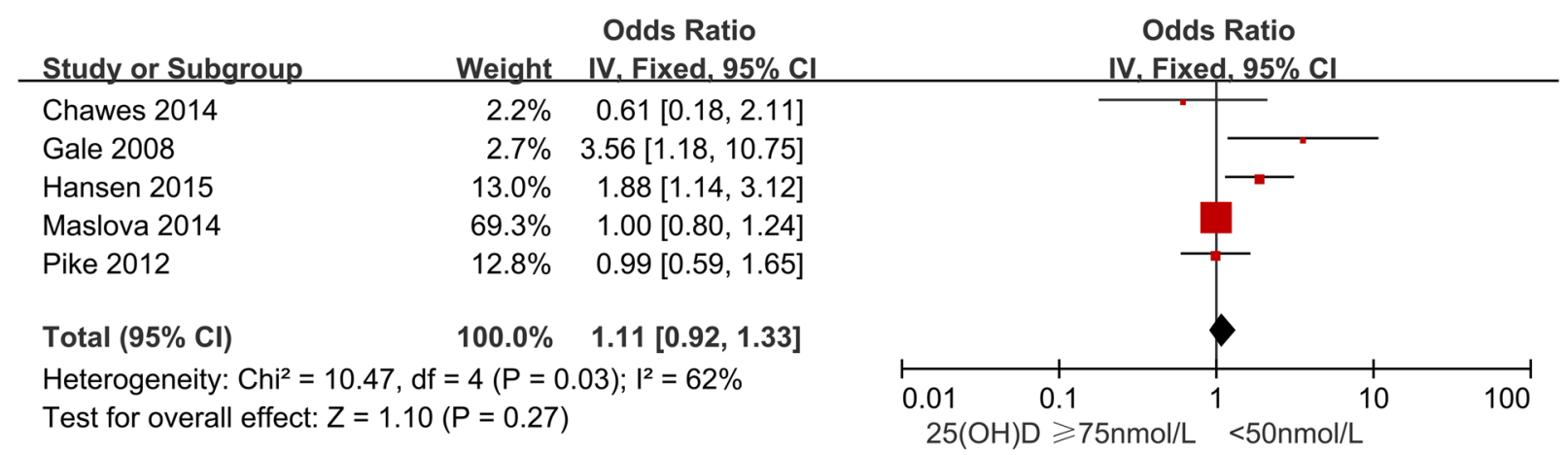

Fig. 2 Study-specific and summary effects of pooled risk estimates of blood vitamin D levels and asthma assessed $>5$ years in cohort studies. a Highest category vs. lowest category of vitamin $D ; \mathbf{b} \geq 75 \mathrm{nmol} / \mathrm{L}$ vs. $<50 \mathrm{nmol} / \mathrm{L}$ 
Table 3 Summary ORs of asthma and early life blood vitamin D levels (highest versus lowest category) in cohort studies, according to selected subgroups

\begin{tabular}{|c|c|c|c|c|c|c|c|c|}
\hline \multicolumn{5}{|l|}{ Age at outcome assessment $>5 y$} & \multicolumn{4}{|c|}{ Age at outcome assessment $\leq 5 y$} \\
\hline Subgroup & $\begin{array}{l}\text { Studies } \\
\text { (Ref. no.) }\end{array}$ & $\begin{array}{l}\text { No. of } \\
\text { studies }\end{array}$ & $\begin{array}{l}\mathrm{OR} \\
(95 \% \mathrm{Cl})\end{array}$ & $1^{2}$ & $\begin{array}{l}\text { Studies } \\
\text { (Ref. no.) }\end{array}$ & $\begin{array}{l}\text { No. of } \\
\text { studies }\end{array}$ & $\begin{array}{l}\mathrm{OR} \\
(95 \% \mathrm{Cl})\end{array}$ & $1^{2}$ \\
\hline \multicolumn{9}{|l|}{ Window of exposure assessment } \\
\hline During pregnancy (mother) & $\begin{array}{l}{[20,22,31-33,36,37} \\
]\end{array}$ & 7 & $\begin{array}{l}0.98 \\
{[0.80,1.20]}\end{array}$ & $49 \%$ & $\begin{array}{l}{[30,32,34,35,} \\
45]\end{array}$ & 5 & $\begin{array}{l}0.79 \\
{[0.63,0.99]}\end{array}$ & $0 \%$ \\
\hline Cord blood & {$[37,41]$} & 2 & $\begin{array}{l}1.03 \\
{[0.60,1.79]}\end{array}$ & $0 \%$ & [23] & 1 & $\begin{array}{l}2.00 \\
{[0.48,8.31]}\end{array}$ & - \\
\hline \multicolumn{9}{|l|}{ Period of outcome assessment } \\
\hline At an end point (e.g. at age 1y) & {$[20,22,31-33,41]$} & 6 & $\begin{array}{l}0.94 \\
{[0.75,1.17]}\end{array}$ & $39 \%$ & {$[30,32,34]$} & 3 & $\begin{array}{l}0.81 \\
{[0.64,1.02]}\end{array}$ & $20 \%$ \\
\hline Up to an end point (e.g. by age $1 y$ ) & {$[32,36,37]$} & 3 & $\begin{array}{l}1.12 \\
{[0.77,1.64]}\end{array}$ & $49 \%$ & {$[23,35,45]$} & 3 & $\begin{array}{l}0.83 \\
{[0.40,1.75]}\end{array}$ & $17 \%$ \\
\hline \multicolumn{9}{|l|}{ Latitude } \\
\hline $\begin{array}{l}\text { Tropics or subtropics (more intense UVR) } \\
\left(\leq 40^{\circ} \mathrm{N} / \mathrm{S}\right)\end{array}$ & {$[31]$} & 1 & $\begin{array}{l}0.58 \\
{[0.29,1.15]}\end{array}$ & - & {$[23,30,35,45]$} & 4 & $\begin{array}{l}0.87 \\
{[0.61,1.24]}\end{array}$ & $0 \%$ \\
\hline Temperate $\left(40-66.5^{\circ} \mathrm{N} / \mathrm{S}\right)$ & $\begin{array}{l}{[20,22,32,33,36,37,41} \\
]\end{array}$ & 7 & $\begin{array}{l}1.01 \\
{[0.82,1.25]}\end{array}$ & $40 \%$ & {$[32,34]$} & 2 & $\begin{array}{l}0.77 \\
{[0.57,1.03]}\end{array}$ & $54 \%$ \\
\hline \multicolumn{9}{|l|}{ Region } \\
\hline Europe & $\begin{array}{l}{[20,22,32,33,36,37,} \\
41]\end{array}$ & 7 & $\begin{array}{l}1.01 \\
{[0.82,1.25]}\end{array}$ & $40 \%$ & {$[30,32,34]$} & 3 & $\begin{array}{l}0.81 \\
{[0.64,1.02]}\end{array}$ & $20 \%$ \\
\hline USA/Canada & & & & & [23] & 1 & $\begin{array}{l}2.00 \\
{[0.48,8.31]}\end{array}$ & - \\
\hline Australia & {$[31]$} & 1 & $\begin{array}{l}0.58 \\
{[0.29,1.15]}\end{array}$ & - & {$[45]$} & 1 & $\begin{array}{l}0.74 \\
{[0.26,2.14]}\end{array}$ & - \\
\hline Asia & & & & & [35] & 1 & $\begin{array}{l}0.40 \\
{[0.09,1.80]}\end{array}$ & - \\
\hline \multicolumn{9}{|l|}{ Adjusted for family history of atopy } \\
\hline No & {$[20,33,36]$} & 3 & $\begin{array}{l}1.14 \\
{[0.88,1.48]}\end{array}$ & $67 \%$ & {$[23,34,35]$} & 3 & $\begin{array}{l}0.70 \\
{[0.50,0.96]}\end{array}$ & $25 \%$ \\
\hline Yes & {$[22,31,32,37,41]$} & 5 & $\begin{array}{l}0.75 \\
{[0.55,1.03]}\end{array}$ & $0 \%$ & {$[30,32,45]$} & 3 & $\begin{array}{l}0.93 \\
{[0.68,1.28]}\end{array}$ & $0 \%$ \\
\hline \multicolumn{9}{|l|}{ Adjusted for seasonality } \\
\hline No & {$[20,22,31]$} & 3 & $\begin{array}{l}0.92[0.62 \\
1.37]\end{array}$ & $69 \%$ & {$[30,45]$} & 2 & $\begin{array}{l}0.87 \\
{[0.60,1.26]}\end{array}$ & $0 \%$ \\
\hline Yes & {$[32,33,36,37,41]$} & 5 & $\begin{array}{l}0.98 \\
{[0.78,1.24]}\end{array}$ & $30 \%$ & {$[23,32,34,35]$} & 4 & $\begin{array}{l}0.78 \\
{[0.59,1.03]}\end{array}$ & $35 \%$ \\
\hline \multicolumn{9}{|l|}{ Adjusted for smoking statues } \\
\hline No & {$[20,22,33]$} & 3 & $\begin{array}{l}1.04 \\
{[0.81,1.33]}\end{array}$ & $52 \%$ & {$[35,45]$} & 2 & $\begin{array}{l}0.60 \\
{[0.25,1.44]}\end{array}$ & $0 \%$ \\
\hline Yes & {$[31,32,36,37,41]$} & 5 & $\begin{array}{l}0.84 \\
{[0.60,1.18]}\end{array}$ & $44 \%$ & {$[23,30,32,34]$} & 4 & $\begin{array}{l}0.83 \\
{[0.66,1.04]}\end{array}$ & $26 \%$ \\
\hline \multicolumn{9}{|l|}{ Outcome assessment } \\
\hline $\begin{array}{l}\text { Maternal report of doctor diagnosed or } \\
\text { medication only }\end{array}$ & {$[22,36,37]$} & 3 & $\begin{array}{l}1.04 \\
{[0.75,1.45]}\end{array}$ & $49 \%$ & {$[23,30,45]$} & 3 & $\begin{array}{l}0.92 \\
{[0.64,1.32]}\end{array}$ & $0 \%$ \\
\hline $\begin{array}{l}\text { Maternal report of doctor diagnosed plus } \\
\text { medication and/or wheeze symptom }\end{array}$ & {$[31-33]$} & 3 & $\begin{array}{l}0.90 \\
{[0.69,1.16]}\end{array}$ & $33 \%$ & {$[32,34,35]$} & 3 & $\begin{array}{l}0.75 \\
{[0.57,1.00]}\end{array}$ & $31 \%$ \\
\hline Doctor diagnosed or medical review & [41] & 1 & $\begin{array}{l}0.61 \\
{[0.18,2.13]}\end{array}$ & - & & & & \\
\hline Other definition & {$[20]$} & 1 & $\begin{array}{l}5.40 \\
{[1.09,26.65]}\end{array}$ & - & & & & \\
\hline
\end{tabular}

Blood 25(OH)D measurement 
Table 3 Summary ORs of asthma and early life blood vitamin D levels (highest versus lowest category) in cohort studies, according to selected subgroups (Continued)

\begin{tabular}{|c|c|c|c|c|c|c|c|c|}
\hline \multicolumn{5}{|c|}{ Age at outcome assessment $>5 y$} & \multicolumn{4}{|c|}{ Age at outcome assessment $\leq 5 \mathrm{y}$} \\
\hline Subgroup & $\begin{array}{l}\text { Studies } \\
\text { (Ref. no.) }\end{array}$ & $\begin{array}{l}\text { No. of } \\
\text { studies }\end{array}$ & $\begin{array}{l}\mathrm{OR} \\
(95 \% \mathrm{Cl})\end{array}$ & $1^{2}$ & $\begin{array}{l}\text { Studies } \\
\text { (Ref. no.) }\end{array}$ & $\begin{array}{l}\text { No. of } \\
\text { studies }\end{array}$ & $\begin{array}{l}\mathrm{OR} \\
(95 \% \mathrm{Cl})\end{array}$ & $1^{2}$ \\
\hline LC-MS/MS (gold standard) & {$[32,33,36,37,41]$} & 5 & $\begin{array}{l}0.98 \\
{[0.78,1.24]}\end{array}$ & $30 \%$ & $\begin{array}{l}{[23,30,32,34,} \\
45]\end{array}$ & 5 & $\begin{array}{l}0.82 \\
{[0.66,1.03]}\end{array}$ & $2 \%$ \\
\hline Other method & {$[20,22,31]$} & 3 & $\begin{array}{l}0.92 \\
{[0.62,1.37]}\end{array}$ & $69 \%$ & {$[35]$} & 1 & $\begin{array}{l}0.40 \\
{[0.09,1.80]}\end{array}$ & - \\
\hline \multicolumn{9}{|l|}{ Risk of bias } \\
\hline Moderate & $\begin{array}{l}{[22,30,32,33,36,} \\
37,41]\end{array}$ & 6 & $\begin{array}{l}0.98 \\
{[0.80,1.21]}\end{array}$ & $13 \%$ & $\begin{array}{l}{[23,30,32,34,} \\
45]\end{array}$ & 5 & $\begin{array}{l}0.82 \\
{[0.66,1.03]}\end{array}$ & $2 \%$ \\
\hline High & {$[20,31]$} & 2 & $\begin{array}{l}1.57 \\
{[0.18,13.80]}\end{array}$ & $84 \%$ & {$[35]$} & 1 & $\begin{array}{l}0.40 \\
{[0.09,1.80]}\end{array}$ & - \\
\hline Overall & $\begin{array}{l}{[20,22,31-33,36} \\
37,41]\end{array}$ & 8 & $\begin{array}{l}0.96 \\
{[0.79,1.18]}\end{array}$ & $43 \%$ & $\begin{array}{l}{[23,30,32,34,} \\
35,45]\end{array}$ & 6 & $\begin{array}{l}0.81 \\
{[0.65,1.01]}\end{array}$ & $0 \%$ \\
\hline
\end{tabular}

ISAAC: The International Study of Asthma and Allergies in Children; LC-MS/MS: liquid chromatography-tandem mass spectrometry; UVR: ultraviolet radiation

\section{a Highest category vs. lowest category of vitamin D}

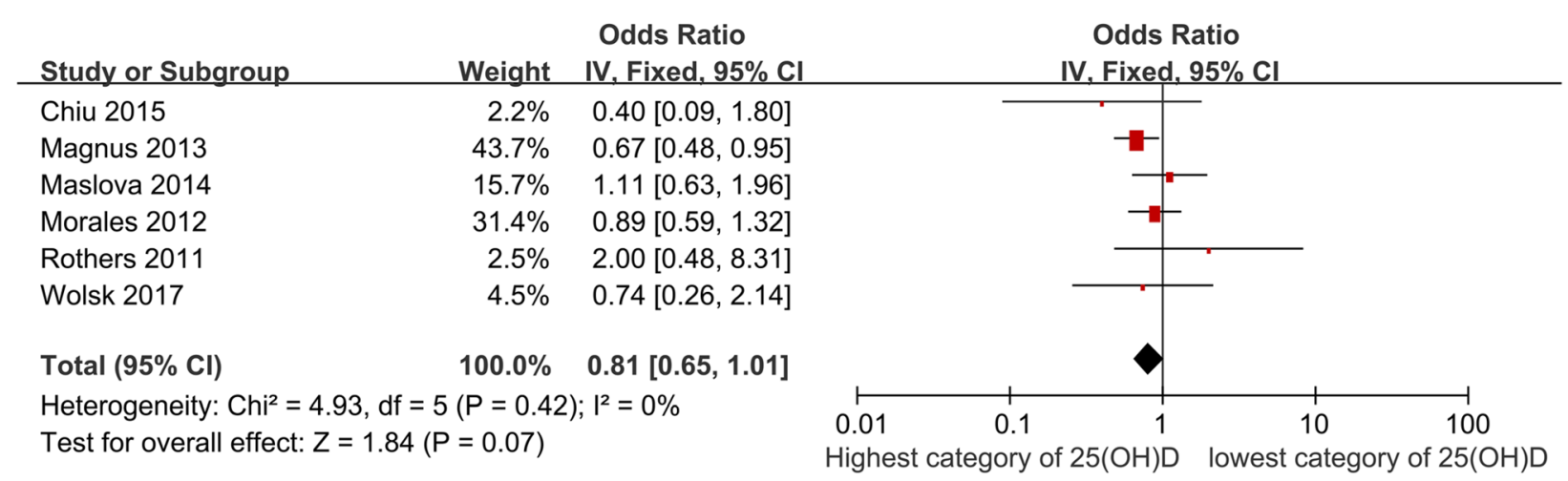

\section{b $\geq 75 \mathrm{nmol} / \mathrm{L}$ vs. $<50 \mathrm{nmol} / \mathrm{L}$}

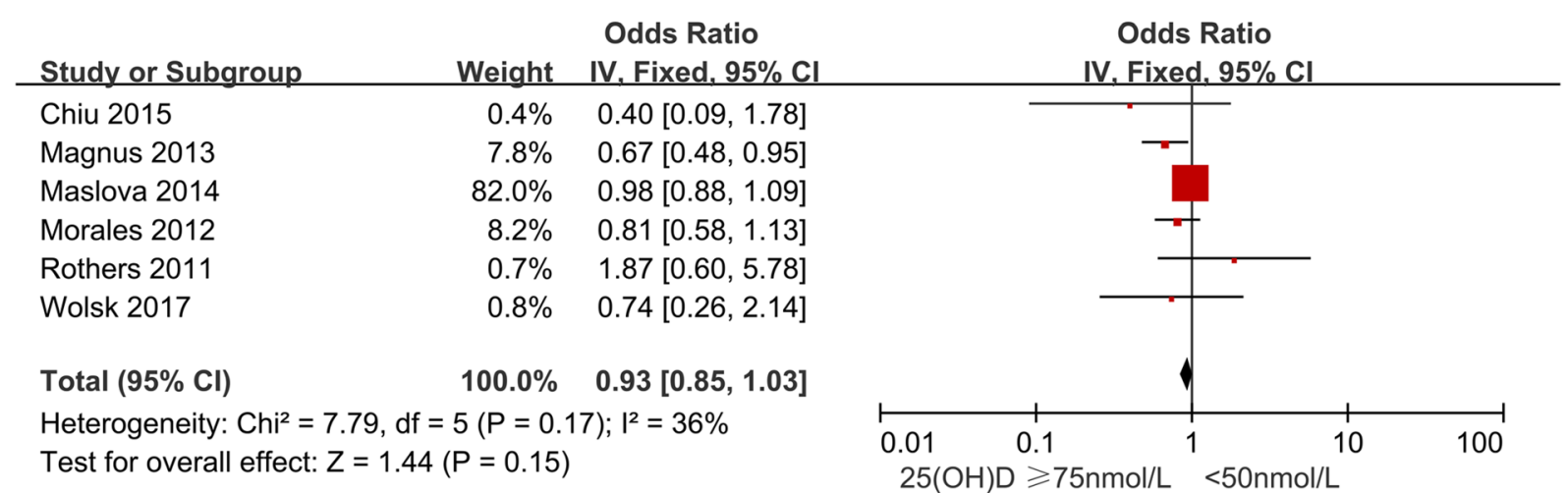

Fig. 3 Study-specific and summary effects of pooled risk estimates of blood vitamin D levels and asthma assessed $\leq 5$ years in cohort studies. a Highest category vs. lowest category of vitamin $D ; \mathbf{b} \geq 75 \mathrm{nmol} / \mathrm{L}$ vs. $<50 \mathrm{nmol} / \mathrm{L}$ 
Table 4 Summary ORs of asthma and early life vitamin D intake (highest versus lowest category) in cohort studies, according to selected subgroups

\begin{tabular}{|c|c|c|c|c|c|c|c|c|}
\hline \multicolumn{5}{|l|}{ Age at outcome assessment $>5 y$} & \multicolumn{4}{|c|}{ Age at outcome assessment $\leq 5 \mathrm{y}$} \\
\hline Subgroup & $\begin{array}{l}\text { Studies } \\
\text { (Ref. no.) }\end{array}$ & $\begin{array}{l}\text { No. of } \\
\text { studies }\end{array}$ & OR $(95 \% \mathrm{Cl})$ & $1^{2}$ & $\begin{array}{l}\text { Studies } \\
\text { (Ref. no.) }\end{array}$ & $\begin{array}{l}\text { No. of } \\
\text { studies }\end{array}$ & OR $(95 \% \mathrm{Cl})$ & $1^{2}$ \\
\hline \multicolumn{9}{|l|}{ Window of exposure assessment } \\
\hline During pregnancy (mother) & {$[50,57]$} & 2 & $\begin{array}{l}1.21 \\
{[0.90,1.62]}\end{array}$ & $64 \%$ & {$[49,53,55,56]$} & 4 & $0.89[0.77,1.04]$ & $68 \%$ \\
\hline During infancy & {$[52]$} & 1 & $\begin{array}{l}0.74 \\
{[0.56,0.96]}\end{array}$ & - & {$[52,54]$} & 2 & $0.95[0.85,1.06]$ & $27 \%$ \\
\hline \multicolumn{9}{|l|}{ Period of outcome assessment } \\
\hline At an end point (e.g. at age 1y) & {$[50,52,57]$} & 3 & $\begin{array}{l}0.90 \\
{[0.56,1.45]}\end{array}$ & $77 \%$ & [54] & 1 & $0.60[0.27,1.31]$ & - \\
\hline Up to an end point (e.g. by age 1y) & {$[50]$} & 1 & $\begin{array}{l}0.60 \\
{[0.32,1.11]}\end{array}$ & - & {$[49,52,53,55,56]$} & 5 & $0.94[0.86,1.02]$ & $60 \%$ \\
\hline \multicolumn{9}{|l|}{ Latitude } \\
\hline $\begin{array}{l}\text { Tropics or subtropics (more intense UVR) } \\
\left(\leq 40^{\circ} \mathrm{N} / \mathrm{S}\right)\end{array}$ & - & - & - & - & [54] & 1 & $0.60[0.27,1.31]$ & - \\
\hline Temperate $\left(40-66.5^{\circ} \mathrm{N} / \mathrm{S}\right)$ & {$[50,52,57]$} & 3 & $\begin{array}{l}0.90 \\
{[0.56,1.45]}\end{array}$ & $77 \%$ & {$[49,52,53,55,56]$} & 5 & $0.94[0.86,1.02]$ & $60 \%$ \\
\hline \multicolumn{9}{|l|}{ Region } \\
\hline Europe & {$[50,52,57]$} & 3 & $\begin{array}{l}0.90 \\
{[0.56,1.45]}\end{array}$ & $77 \%$ & {$[49,52,53,56]$} & 4 & $0.96[0.88,1.05]$ & $47 \%$ \\
\hline USA/Canada & & & & & {$[55]$} & 1 & $0.65[0.46,0.93]$ & - \\
\hline Asia & & & & & [54] & 1 & $0.60[0.27,1.31]$ & - \\
\hline \multicolumn{9}{|l|}{ Adjusted for family history of atopy } \\
\hline No & {$[57]$} & 1 & $\begin{array}{l}1.33 \\
{[0.97,1.82]}\end{array}$ & - & {$[55]$} & 1 & $0.65[0.46,0.93]$ & - \\
\hline Yes & {$[50,52]$} & 2 & $\begin{array}{l}0.73 \\
{[0.56,0.94]}\end{array}$ & $0 \%$ & {$[49,52-54,56]$} & 5 & $0.95[0.87,1.04]$ & $43 \%$ \\
\hline \multicolumn{9}{|l|}{ Adjusted for seasonality } \\
\hline No & {$[57]$} & 1 & $\begin{array}{l}1.33 \\
{[0.97,1.82]}\end{array}$ & - & {$[53-56]$} & 4 & $0.64[0.48,0.86]$ & $29 \%$ \\
\hline Yes & {$[50,52]$} & 2 & $\begin{array}{l}0.73 \\
{[0.56,0.94]}\end{array}$ & $0 \%$ & {$[49,52]$} & 2 & $0.97[0.88,1.06]$ & $0 \%$ \\
\hline \multicolumn{9}{|l|}{ Adjusted for smoking statues } \\
\hline No & {$[57]$} & 1 & $\begin{array}{l}1.33 \\
{[0.97,1.82]}\end{array}$ & - & {$[56]$} & 1 & $3.16[0.61,16.32]$ & - \\
\hline Yes & {$[50,52]$} & 2 & $\begin{array}{l}0.73 \\
{[0.56,0.94]}\end{array}$ & $0 \%$ & {$[49,52-55]$} & 5 & $0.93[0.85,1.01]$ & $56 \%$ \\
\hline \multicolumn{9}{|l|}{ Outcome assessment } \\
\hline $\begin{array}{l}\text { Maternal report of doctor diagnosed or } \\
\text { medication only }\end{array}$ & {$[50]$} & 1 & $\begin{array}{l}0.60 \\
{[0.32,1.11]}\end{array}$ & - & {$[52,53]$} & 2 & $0.94[0.85,1.05]$ & $71 \%$ \\
\hline $\begin{array}{l}\text { Maternal report of doctor diagnosed plus } \\
\text { medication and/or wheeze symptom }\end{array}$ & {$[50,52,57]$} & 3 & $\begin{array}{l}0.90 \\
{[0.56,1.45]}\end{array}$ & $77 \%$ & {$[49,55]$} & 2 & $0.82[0.55,1.24]$ & $77 \%$ \\
\hline Doctor diagnosed or medical review & - & - & - & - & {$[54]$} & 1 & $0.60[0.27,1.31]$ & - \\
\hline Based on ISAAC questionnaire & - & - & - & - & {$[56]$} & 1 & $3.16[0.61,16.32]$ & - \\
\hline \multicolumn{9}{|l|}{ Risk of bias } \\
\hline Moderate & {$[52,57]$} & 2 & $\begin{array}{l}0.98 \\
{[0.55,1.75]}\end{array}$ & $87 \%$ & {$[49,52,54,56]$} & 4 & $0.96[0.88,1.06]$ & $14 \%$ \\
\hline High & {$[50]$} & 1 & $\begin{array}{l}0.63 \\
{[0.28,1.44]}\end{array}$ & - & {$[53,55]$} & 2 & $0.61[0.45,0.84]$ & $0 \%$ \\
\hline Overall & {$[50,52,57]$} & 3 & $\begin{array}{l}0.90 \\
{[0.56,1.45]}\end{array}$ & $77 \%$ & {$[49,52-56]$} & 6 & $0.93[0.85,1.02]$ & $56 \%$ \\
\hline
\end{tabular}


significant associations in the stratified analyses for studies that had more robust methodology (adjusting for further confounders, e.g. family history of atopy, seasonality, smoking status; blood calcifediol measured by the gold standard LC-MS/MS; studies with lower risk of bias) (Table 3). Six eligible cohort studies [38, 39, 43, 44, 46, 47] analyzed blood vitamin $\mathrm{D}$ as a continuous variable and we found no association with childhood asthma.

There was no association between vitamin D intake and asthma at $\leq 5$ years (6 cohorts, 35,257 participants) or $>5$ years ( 3 cohorts, 32,023 participants) (Table 4). A significant inverse association between vitamin $\mathrm{D}$ intake and asthma at $\leq 5$ years of age emerged when we restricted our analysis to studies that did not adjust for seasonality or had high risk of bias, with low heterogeneity within these subgroups; and between vitamin D intake and asthma at $>5$ years of age when we restricted to studies that adjusted for family history of atopy, seasonality or smoking status, or those that assessed outcome in childhood (OR $0.73,95 \%$ CI $0.56,0.94, \mathrm{I}^{2}=0 \%$, 25,301 participants from 2 studies, for highest vs. lowest) (Table 4). The number needed to treat to prevent one case of asthma was 39 (range 25-93) via maternal vitamin D supplementation (Additional file 1: Table S2).

Studies that measured blood vitamin D concentrations generally had a low risk of bias except for attrition bias due to incomplete outcome data, confounding bias, and detection bias in the assessment of prognostic factors (Additional file 1: Figures S2 and S3). Several studies that assessed vitamin D intake had a high risk of detection bias due to recall of vitamin $\mathrm{D}$ intake, confounding bias, and attrition bias due to incomplete outcome data. (Additional file 1: Figures S4 and S5). No evidence of publication bias was found from the funnel plots or Egger's tests for blood $(P=0.963$ for asthma $>5$ years and $P=0.655$ for asthma $\leq 5$ years $)$ and intake $(P=0.913$ for asthma $>5$ years and $P=0.410$ for asthma $\leq 5$ years) (Additional file 1: Table S3).

\section{Early life vitamin D status and wheeze}

All three RCTs [26-28] used wheeze as primary outcome (one combined with asthma [28]). Two RCTs found no definitive effect of vitamin $\mathrm{D}$ supplementation during pregnancy on wheeze among offspring by 3 years of age

\section{a Highest category vs. lowest category of vitamin D}

\begin{tabular}{lrcccc} 
Study or Subgroup & Weight & $\begin{array}{c}\text { Odds Ratio } \\
\text { IV, Fixed, 95\% Cl }\end{array}$ & Odds Ratio \\
\hline IV, Fixed, 95\% Cl
\end{tabular}

b $\geq 75 \mathrm{nmol} / \mathrm{L}$ vs. $<50 \mathrm{nmol} / \mathrm{L}$

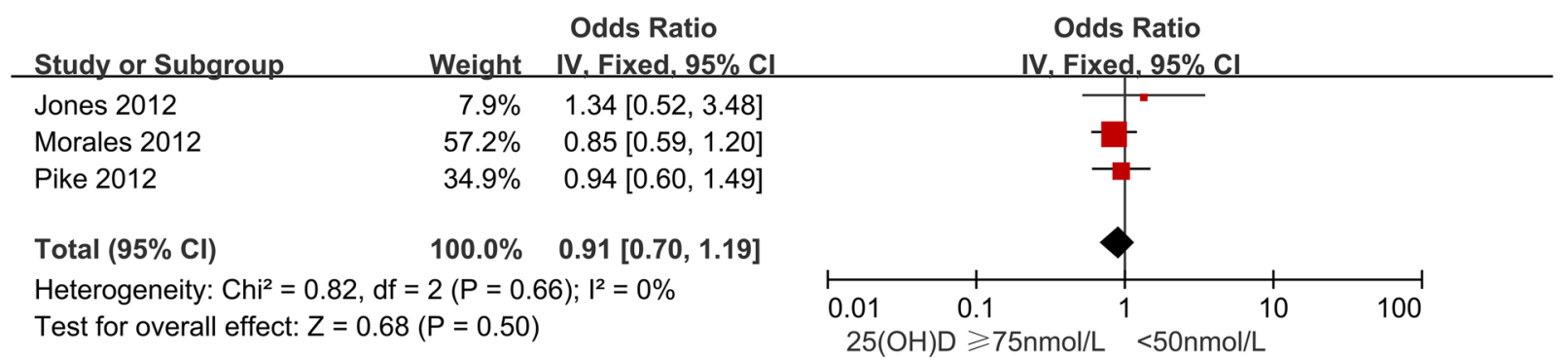

Fig. 4 Study-specific and summary effects of pooled risk estimates of blood vitamin D levels and wheeze in cohort studies. a Highest category vs. lowest category of vitamin $\mathrm{D} ; \mathbf{b} \geq 75 \mathrm{nmol} / \mathrm{L}$ vs. $<50 \mathrm{nmol} / \mathrm{L}$ 
(OR 0.86; 95\% CI 0.49, 1.50; HR 0.76; 95\% CI 0.52, 1.12) and one found a borderline statistical significance association (HR 0.8; 95\% CI 0.6, 1.0). We did not perform pooled analysis for wheeze because of the major differences in intervention. Risks of performance and attrition bias were considered to be high due to missing outcome data and the lack of blinding in the trial conducted by Goldring et al. [26] (Additional file 1: Figure S1).

We did not observe significant association between maternal vitamin $\mathrm{D}$ concentrations during pregnancy and offspring wheeze in cohort studies $(8123$ participants from 8 studies, OR 0.91, 95\% CI 0.76, 1.09 for highest vs. lowest levels, $\mathrm{I}^{2}=25 \%$; 2324 participants from 3 studies, OR 0.91, 95\% CI 0.70, 1.19 for $\geq 75 \mathrm{nmol} / \mathrm{L}$ vs. $<50 \mathrm{nmol} / \mathrm{L}, \mathrm{I}^{2}=0 \%$ ) (Fig. 4). Similar results were observed in stratified analyses (Table 5). Within-group inconsistency disappeared or was attenuated in studies that measured vitamin D intake during pregnancy or adjusted for family history of atopy or seasonality or blood calcifediol measured by LC-MS/MS or studies with moderate risk of bias. The overall fixed-effects OR of wheeze relating to maternal vitamin $\mathrm{D}$ intake was 0.66 (95\% CI 0.53, 0.82; 5238 participants from 6 studies, $\mathrm{I}^{2}=52 \%$, Table 5). The association remained statistically significant when restricting to studies that: assessed outcome at or before 3 years of age and after 3 years of age, were conducted in a temperate area, adjusted for family history of atopy or seasonality, and those that had lower risk of bias. The Baiz paper [38] did not find an association in early transient, late onset or persistent wheeze, while Camargo et al. [39] reported a significant protective effect by 5 years of age (OR 0.95, 95\% CI 0.91 ,

Table 5 Summary ORs of wheeze and vitamin D status (highest versus lowest category) in cohort studies, according to selected subgroups

\begin{tabular}{|c|c|c|c|c|c|c|c|c|}
\hline \multicolumn{5}{|l|}{ Early life blood vitamin D levels } & \multicolumn{4}{|c|}{ Early life vitamin D intake } \\
\hline Subgroup & $\begin{array}{l}\text { Studies } \\
\text { (Ref. no.) }\end{array}$ & $\begin{array}{l}\text { No. of } \\
\text { studies }\end{array}$ & $\begin{array}{l}\mathrm{OR} \\
(95 \% \mathrm{Cl})\end{array}$ & $1^{2}$ & $\begin{array}{l}\text { Studies } \\
\text { (Ref. no.) }\end{array}$ & $\begin{array}{l}\text { No. of } \\
\text { studies }\end{array}$ & $\begin{array}{l}\text { OR } \\
(95 \% \mathrm{Cl})\end{array}$ & $1^{2}$ \\
\hline \multicolumn{9}{|l|}{ Window of exposure assessment } \\
\hline During pregnancy (mother) & {$[22,30,31,33,37]$} & 5 & $0.93[0.76,1.12]$ & $9 \%$ & $\begin{array}{l}{[21,48,49,51,54,} \\
55]\end{array}$ & 6 & $0.66[0.53,0.82]$ & $52 \%$ \\
\hline Cord blood & {$[21,40,42]$} & 3 & $0.83[0.51,1.35]$ & $58 \%$ & - & - & - & - \\
\hline During infancy (infant) & {$[42]$} & 1 & $1.88[0.89,3.98]$ & & & & & \\
\hline \multicolumn{9}{|l|}{ Age at outcome assessment } \\
\hline$\leq 3 y$ & {$[21,29,30,37,40,42]$} & 6 & $0.93[0.77,1.12]$ & $26 \%$ & {$[21,48,51,54]$} & 4 & $0.68[0.53,0.88]$ & $63 \%$ \\
\hline$>3 y$ & {$[22,30,31,33,37]$} & 5 & $1.09[0.89,1.33]$ & $11 \%$ & {$[49,55]$} & 2 & $0.57[0.35,0.93]$ & $48 \%$ \\
\hline \multicolumn{9}{|l|}{ Latitude } \\
\hline $\begin{array}{l}\text { Tropics or subtropics } \\
\text { (more intense UVR) }\left(\leq 40^{\circ} \mathrm{N} / \mathrm{S}\right)\end{array}$ & {$[21,30,31]$} & 3 & $0.83[0.60,1.14]$ & $47 \%$ & {$[21,48,54]$} & 3 & $0.81[0.61,1.07]$ & $7 \%$ \\
\hline Temperate $\left(40-66.5^{\circ} \mathrm{N} / \mathrm{S}\right)$ & {$[22,33,37,40,42]$} & 5 & $0.95[0.77,1.18]$ & $21 \%$ & {$[49,51,55]$} & 3 & $0.47[0.33,0.68]$ & $35 \%$ \\
\hline \multicolumn{9}{|l|}{ Adjusted for family history of atopy } \\
\hline No & {$[40]$} & 1 & $0.36[0.14,0.89]$ & - & {$[21,55]$} & 2 & $0.84[0.47,1.49]$ & $46 \%$ \\
\hline Yes & $\begin{array}{l}{[21,22,30,31,33,37,} \\
42]\end{array}$ & 7 & $0.95[0.79,1.14]$ & $0 \%$ & {$[48,49,51,54]$} & 4 & $0.63[0.49,0.80]$ & $61 \%$ \\
\hline \multicolumn{9}{|l|}{ Adjusted for seasonality } \\
\hline No & {$[22,30,31,40]$} & 4 & $0.78[0.60,1.01]$ & $51 \%$ & {$[21,48,54,55]$} & 4 & $0.79[0.61,1.03]$ & $0 \%$ \\
\hline Yes & {$[21,33,37,42]$} & 4 & $1.05[0.82,1.33]$ & $0 \%$ & {$[49,51]$} & 2 & $0.37[0.24,0.58]$ & $0 \%$ \\
\hline \multicolumn{9}{|l|}{ Blood 25(OH)D measurement } \\
\hline LC-MS/MS (gold standard) & {$[21,30,33,37,40,42]$} & 6 & $0.97[0.79,1.19]$ & $10 \%$ & - & - & - & - \\
\hline Other methods & {$[22,31]$} & 2 & $0.76[0.53,1.10]$ & $60 \%$ & - & - & - & - \\
\hline \multicolumn{9}{|l|}{ Risk of bias } \\
\hline Moderate & {$[22,30,33,37]$} & 4 & $0.99[0.81,1.21]$ & $0 \%$ & {$[48,49,51,54]$} & 4 & $0.63[0.49,0.80]$ & $61 \%$ \\
\hline High & {$[21,31,40,42]$} & 4 & $0.69[0.47,1.01]$ & $52 \%$ & {$[21,55]$} & 2 & $0.84[0.47,1.49]$ & $46 \%$ \\
\hline Overall & $\begin{array}{l}{[21,22,30,31,33,37,} \\
40,42]\end{array}$ & 8 & $0.91[0.76,1.09]$ & $25 \%$ & $\begin{array}{l}{[21,48,49,51} \\
54,55]\end{array}$ & 6 & $0.66[0.53,0.82]$ & $52 \%$ \\
\hline
\end{tabular}


0.99), but this has not been consistent in earlier time windows reported in the studies by Camargo et al. [39] and Visness et al. [44]

The 12 cohort studies that investigated blood vitamin D levels and wheeze were evaluated to have low risk of bias, in terms of participant selection, exposure assessment, and window of outcome assessment and co-intervention. Some studies had high or unclear risk of confounding bias, detection bias and attrition bias (Additional file 1: Figure S6, a and b). Overall, the studies on vitamin $\mathrm{D}$ intake had higher risk of bias, due to their inadequacy in exposure assessment, assessment and adjustment for prognostic factors, and outcome follow-up (Additional file 1: Figure S7, a and b). There was no evidence of publication bias from the funnel plots or Egger's tests for blood $(P=0.772)$ and intake $(P=0.954)$ (Additional file 1 : Table S3).

\section{Discussion}

This meta-analysis of cohort studies found no statistically significant association between vitamin D levels in maternal or cord blood or intake in early life and asthma either at $>5$ or $\leq 5$ years of age, with no evidence of publication bias. Of the cohort studies included, only two studies assessed asthma in adulthood [36, 57]. The exclusion of these two studies did not change the pooled estimate for blood vitamin D levels, while a significant inverse association between early life vitamin intake and childhood asthma at $>5$ years emerged. We found no association between early life vitamin D level and risk of wheeze in later life. On the other hand, findings from cohort studies seemed to suggest a lower risk of wheeze associated with maternal vitamin $\mathrm{D}$ intake during pregnancy.

Variations in the intervention and outcome definition in three identified RCTs made it difficult to pool the effect size for wheeze or asthma associated with vitamin D supplementation during pregnancy. Two more recent trials [27, 28] with lower risk of bias also found non-statistically significant benefit for persistent/recurrent wheeze or asthma in the first 3 years of life. The results of RCTs were in line with the results of our meta-analysis of cohort studies, where we found non-significant trends of vitamin $\mathrm{D}$ supplementation for preventing offspring asthma at $\leq 5$ years. However, our findings from cohort studies also suggest that early-life vitamin D intake may have an effect on childhood asthma at age $>5$ years and childhood wheeze. Longer follow-up time for assessing the effect of vitamin $\mathrm{D}$ intake against asthma is required in future RCTs to confirm these results [58].

There are two opposing views regarding the relationship between vitamin D exposure and asthma. One school of thought, based on growing epidemiological evidence, hypothesized a link between vitamin D deficiency in early life and development of asthma and other allergic diseases [59], thought to have begun as sunlight exposure decreased with industrialization and urbanization. Others argued, however, that the current asthma and allergic disease pandemic might have been the consequence of widespread vitamin $\mathrm{D}$ supplementation in food, backed by some historical evidence [60]. Coincidentally, both low and high levels of vitamin $\mathrm{D}$ were reported to be related to asthma, in a U-shaped manner [32]. Another study by Rothers et al. also found that both low $(<50 \mathrm{nmol} / \mathrm{L})$ and high $(\geq 100 \mathrm{nmol} / \mathrm{L})$ levels of cord blood calcifediol were associated with increased IgE, which was related to subsequent risk of wheeze/asthma [23]. In the present meta-analysis, the inconsistent results from studies assessing vitamin D either in blood or intake for childhood asthma/wheeze risk may be explained by the previously reported non-linear association between blood vitamin $\mathrm{D}_{3}$ and childhood asthma, which tends to be non-statistically significant by comparing the highest with lowest vitamin D levels [32]. However, the dichotomized exposure in the present study limited the exploration of non-linear association.

The sources of heterogeneity, as indicated by the results from stratified analyses were: window of exposure assessment, whether family history of atopy or seasonality have been adjusted for, definition of outcome, risk of bias, and the method of assessing blood calcifediol. As suggested by Autier et al. [61], vitamin D level could be a biomarker and proxy for overall health and wellbeing of mothers and infants. However, as most of the cohort studies included did not adjust for health status of the participants, we were unable to exclude such potential confounding. In most of the studies included, tobacco smoking was adjusted for in the analysis, but other important confounders including family history of atopic diseases, seasonal variations in blood vitamin D levels, sedentary lifestyle and obesity [62-64] were less commonly considered. Nevertheless, results from our sensitivity analysis on studies reporting blood vitamin D level, which included only studies that adjusted for family history and seasonal variations, showed no material difference in the summary estimates compared with our main findings. Three of the cohort studies [35, 42, 47] measured serum calcifediol in both prenatal and postnatal periods, but these studies analyzed the associations between vitamin $\mathrm{D}$ measured at different time points with outcome separately and failed to explore the effect of persistent vitamin D deficiency on asthma. While the half-life of serum calcifediol is only about two weeks [65], it is possible that such misclassification in vitamin $\mathrm{D}$ status measured at only one time point could have biased the findings towards the null. A recent study conducted by Hollams et al. used mixed-effects logistic 
regression and found no longitudinal association between calcifediol concentrations as a continuous variable and asthma from 3 to 10 years of age [46].

On the issue of case definition, ascertaining childhood asthma in epidemiological studies has been challenging given the lack of definitive diagnostic criteria [66, 67]. Most studies defined asthma by either maternal report of doctor-diagnosed asthma, asthma medications use, or a combination of doctor-diagnosed asthma and either asthma medication or wheezing symptoms within the past year. Although not without caveats, such as under-reporting both diagnosed and undiagnosed conditions [68] and variable agreement with clinical records [67], these definitions have been widely used in research [69]. In two studies asthma definition was not specified, adding further variability to outcome assessment and increased heterogeneity in our pooled analyses.

In general, studies investigating blood vitamin D levels had relatively low risk of bias in most domains except for confounding, detection, and attrition biases, whereas studies that used food frequency questionnaire to quantify vitamin $\mathrm{D}$ intake were at risk of misclassification bias as pregnant women often had to recall their dietary history from a few months previous.. Apart from detection bias, most vitamin $\mathrm{D}$ intake studies were also at risk of attrition bias for their inadequate follow-up duration or unknown difference between missing and available outcome data, as well as confounding bias in some studies which did not include major confounders. In addition to variation over time, different methods of blood vitamin $\mathrm{D}$ level quantification employed in different studies might also introduce inter-study variability. In the stratified analysis, we compared the risk estimates derived from studies that used the gold standard LC-MS/MS with those using other methods and found no material difference between the pooled estimates. Studies that investigated antenatal vitamin D intake and asthma at $>5$ years of age tended to be small and their inclusion in the meta-analysis may explain the weak evidence observed.

Compared to previous related systematic reviews [11-13, 70], we have identified more cohort studies and included two recently published RCTs in the analysis. Only six cohort studies $[20,22,23,30,33,39]$ measuring maternal serum calcifediol during pregnancy or from cord blood and asthma risk were identified by Cassim et al. [70], but we have included 13 more studies [31, 32, 34-38, 41, 43-47], plus an additional 13 cohort studies [21, 22, 29-31, 33, 37-40, 42, $44,46]$ to explore the association between calcifediol level in maternal blood or cord blood and wheeze risk. As a consequence of our more comprehensive approach in including various exposure metrics (intake and blood level, both maternal and in infant) in the search strategy and a critical risk of bias assessment using the risk of bias tool recommended by the Cochrane Collaboration [17, 18], we were able to demonstrate the existence of heterogeneity between studies in the findings, which lead us to a more conservative conclusion. In addition, we further studied the association between antenatal or early postnatal vitamin D level and asthma and wheeze using commonly accepted clinical cut-off values. We found no difference of risk of asthma or wheeze between sufficient and deficient vitamin D level groups, implying the optimal threshold for bone health may not necessarily be applicable for the respiratory and immune systems.

One of the limitations of this work is that we were unable to retrieve sufficient information for calculating pooled estimates from six eligible studies that analyzed vitamin $\mathrm{D}$ level as a continuous variable. We were able to identify one study that has examined infant blood vitamin D levels and their relationship to wheeze but none for asthma. Studies investigating the association between infant vitamin D levels and risk of childhood asthma should be encouraged, with designs that minimize detection, confounding, and follow-up biases.

\section{Conclusions}

The pooled estimates from cohort studies show no association between antenatal blood vitamin D level and asthma/ wheeze in later life. On the other hand, although the interventional studies found a non-statistically significant benefit for asthma/wheeze in first 3 years of life, the pooled estimates from cohort studies suggest that early life vitamin D intake may have an effect on childhood asthma $>5$ years or childhood wheeze. Should that be the case, this may point to a cost-effective intervention for childhood asthma. Further trials with enough power and longer follow-up time should be conducted to confirm the results.

\section{Additional file}

Additional file 1: Supplementary Materials. (DOCX 290 kb).

\section{Abbreviations}

BMI: body mass index; Cls: confidence intervals; ELISA: enzyme-linked immunosorbent assay; FFQ: food frequency questionnaire; HPLC: high performance liquid chromatography; HR: hazard ratio; IOM: Institute of Medicine; ISAAC: The International Study of Asthma and Allergies in Children; LC-MS/MS: liquid chromatography-tandem mass spectrometry; MOOSE: Metaanalysis of Observational Studies in Epidemiology; OR: odds ratio; PRISMA: Preferred Reporting Items for Systematic Reviews and Meta-Analyses; $\mathrm{RCT}$ : randomized controlled trial; RR: relative risk; UVR: ultraviolet radiation

\section{Acknowledgements}

The authors would like to thank Professor Susan L. Prescott (Centre for Child Health Research, University of Western Australia, Perth, Australia), Dr. Anderson P. Jones (Centre for Child Health Research, University of Western Australia, Perth, Australia), Professor Anne L. Wright (Arizona Respiratory Center, at the University of Arizona, Tucson, AZ), Dr. Debra A. Stern (Arizona Respiratory Center, at the University of Arizona, Tucson, AZ), Dr. Jane SA Lucas (Clinical and Experimental Sciences Academic Unit, University of Southampton Faculty of Medicine, Southampton, UK), Dr. Katharine C Pike (Clinical and Experimental Sciences Academic Unit, University of Southampton Faculty of Medicine, Southampton, UK), Dr. Catharine R Gale (MRC Epidemiology Resource Centre (University of Southampton), 
Southampton General Hospital, Southampton) for re-analyzing and sending the data requested from their manuscripts. We also thank Professor Carlos A Camargo, Jr., Dr. Andrew Wills and Dr. Bo L. Chawes for clarifying our queries relating to their manuscripts and Mr. lan Henderson for helping the language editing of the manuscript.

\section{Funding}

This work was supported by the Guangzhou Science and Technology Bureau, Guangzhou, China (2012 J5100038, 201807010086). The funders had no role in study design, data collection and analysis, decision to publish, interpretation of data and preparation of the manuscript.

\section{Availability of data and materials}

We declare that the data supporting the conclusions of this article are described in the article.

\section{Authors' contributions}

$X Q, H X, K C$ and $S S$ acquired funding. $X Q, S S, K C$ and $K L$ conceived the study. $\mathrm{SS}$ and $\mathrm{KL}$ developed the protocol of the systematic review and conducted the electronic database searches. SS and WX screened titles and abstracts, extracted relevant data, performed the quality grading and drafted the manuscript. SS, JL, JH, and YM performed statistical analysis. SS wrote the manuscript and $\mathrm{KL}$ revised the manuscript. $\mathrm{KC}, \mathrm{XQ}$ and $\mathrm{HX}$ contributed to the interpretation of the findings. All authors reviewed and approved the final manuscript.

\section{Ethics approval and consent to participate}

Not applicable.

\section{Consent for publication}

Not applicable.

\section{Competing interests}

The authors declare that they have no competing interests.

\section{Publisher's Note}

Springer Nature remains neutral with regard to jurisdictional claims in published maps and institutional affiliations.

\section{Author details}

'Division of Birth Cohort Study, Guangzhou Women and Children's Medical Center, Guangzhou Medical University, 9 Junsui Road, Zhujiang Newtown, Tianhe District, Guangzhou 510623, China. ${ }^{2}$ Department of Pediatric Surgery, Guangzhou Women and Children's Medical Center, Guangzhou Medical University, Guangzhou, China. ${ }^{3}$ Institute of Applied Health Research, University of Birmingham, Birmingham, UK. ${ }^{4}$ Clinical Trial Service Unit and Epidemiological Studies Unit, Nuffield Department of Population Health, University of Oxford, Oxford, UK.

\section{Received: 14 February 2017 Accepted: 25 June 2018}

Published online: 20 July 2018

\section{References}

1. Eder W, Ege MJ, von Mutius E. The asthma epidemic. N Engl J Med. 2006; 355(21):2226-35.

2. Song WJ, Kang MG, Chang YS, Cho SH. Epidemiology of adult asthma in Asia: toward a better understanding. Asia Pac Allergy. 2014;4(2):75-85.

3. Akinbami $\amalg$, Moorman JE, Bailey C, Zahran HS, King M, Johnson CA, Liu X. Trends in asthma prevalence, health care use, and mortality in the United States, 2001-2010. NCHS data brief, no 94. Hyattsville, MD: National Center for Health Statistics. 2012(94):1-8.

4. Holick MF. Vitamin D deficiency. N Engl J Med. 2007;357(3):266-81.

5. Halicioglu O, Aksit S, Koc F, Akman SA, Albudak E, Yaprak I, Coker I, Colak A, Ozturk C, Gulec ES. Vitamin D deficiency in pregnant women and their neonates in spring time in western Turkey. Paediatr Perinat Epidemiol. 2012; 26(1):53-60.

6. Salle BL, Delvin EE, Lapillonne A, Bishop NJ, Glorieux FH. Perinatal metabolism of vitamin D. Am J Clin Nutr. 2000;71(5 Suppl):1317S-24S.

7. Vandevijvere S, Amsalkhir S, Van Oyen H, Moreno-Reyes R. High prevalence of vitamin $\mathrm{D}$ deficiency in pregnant women: a national cross-sectional survey. PLoS One. 2012;7(8):e43868.
8. Khuri-Bulos N, Lang RD, Blevins M, Kudyba K, Lawrence L, Davidson M Faouri S, Halasa NB. Vitamin D deficiency among newborns in Amman, Jordan. Glob J Health Sci. 2014;6(1):162-71.

9. Vieth Streym S, Kristine Moller U, Rejnmark L, Heickendorff L, Mosekilde L, Vestergaard P. Maternal and infant vitamin D status during the first 9 months of infant life-a cohort study. Eur J Clin Nutr. 2013;67(10):1022-8.

10. Bozzetto S, Carraro S, Giordano G, Boner A, Baraldi E. Asthma, allergy and respiratory infections: the vitamin D hypothesis. Allergy. 2012;67(1):10-7.

11. Nurmatov $U$, Devereux $G$, Sheikh A. Nutrients and foods for the primary prevention of asthma and allergy: systematic review and meta-analysis. J Allergy Clin Immunol. 2011;127(3):724-733 e721-730.

12. Harvey NC, Holroyd C, Ntani G, Javaid K, Cooper P, Moon R, Cole Z, Tinati T, Godfrey K, Dennison E, et al. Vitamin D supplementation in pregnancy: a systematic review. Health Technol Assess. 2014;18(45):1-190.

13. Beckhaus AA, Garcia-Marcos L, Forno E, Pacheco-Gonzalez RM, Celedon JC, Castro-Rodriguez JA. Maternal nutrition during pregnancy and risk of asthma, wheeze, and atopic diseases during childhood: a systematic review and meta-analysis. Allergy. 2015;70(12):1588-604

14. Stroup DF, Berlin JA, Morton SC, Olkin I, Williamson GD, Rennie D, Moher D, Becker BJ, Sipe TA, Thacker SB. Meta-analysis of observational studies in epidemiology: a proposal for reporting. Meta-analysis of observational studies in epidemiology (MOOSE) group. JAMA. 2000;283(15):2008-12.

15. Moher D, Liberati A, Tetzlaff J, Altman DG. Preferred reporting items for systematic reviews and meta-analyses: the PRISMA statement. J Clin Epidemiol. 2009;62(10):1006-12.

16. Shen SY, Xiao WQ, Lu JH, Lam KB, Qiu X, Cheng KK. Maternal and infant vitamin D status in relation to asthma and allergic diseases: a systematic review and meta-analysis. 2013, http://www.crd.york.ac.uk/prospero/display_ record.asp?ID=CRD42013005559\#.U458kySS1dg. Accessed August, 2017.

17. Higgins JPT, Altman DG, JAC S. Chapter 8 Assessing risk of bias in included studies. In: JPT H, Green S, editors. Cochrane Handbookfor systematic reviews of interventions. London: Cochrane Collaboration; 2011.

18. Cochrane. Tool to Assess Risk of Bias in Cohort Studies. 2013; http://methods cochrane.org/bias/sites/methods.cochrane.org.bias/files/public/uploads/ Tool\%20to\%20Assess\%20Risk\%20of\%20Bias\%20in\%20Cohort\%20Studies.pdf. Accessed August, 2017.

19. Ross AC, Manson JE, Abrams SA, Aloia JF, Brannon PM, Clinton SK, DurazoArvizu RA, Gallagher JC, Gallo RL, Jones G, et al. The 2011 report on dietary reference intakes for calcium and vitamin $D$ from the Institute of Medicine: what clinicians need to know. J Clin Endocrinol Metab. 2011;96(1):53-8.

20. Gale CR, Robinson SM, Harvey NC, Javaid MK, Jiang B, Martyn CN, Godfrey KM, Cooper C. Maternal vitamin D status during pregnancy and child outcomes. Eur J Clin Nutr. 2008;62(1):68-77.

21. Jones AP, Palmer D, Zhang G, Prescott SL. Cord blood 25-hydroxyvitamin D3 and allergic disease during infancy. Pediatrics. 2012;130(5):e1128-35.

22. Pike KC, Inskip HM, Robinson S, Lucas JS, Cooper C, Harvey NC, Godfrey KM, Roberts G. Maternal late-pregnancy serum 25 -hydroxyvitamin D in relation to childhood wheeze and atopic outcomes. Thorax. 2012;67(11):950-6.

23. Rothers J, Wright AL, Stern DA, Halonen M, Camargo Jr CA: Cord blood 25hydroxyvitamin D levels are associated with aeroallergen sensitization in children from Tucson, Arizona. J Allergy Clin Immunol 2011, 128(5):1093-1099.e1095.

24. Cates CJ. Simpson's paradox and calculation of number needed to treat from meta-analysis. BMC Med Res Methodol. 2002;2:3.

25. Cochrane. Revman 5. 2014; http://community.cochrane.org/tools/reviewproduction-tools/revman-5. Accessed August, 2017.

26. Goldring ST, Griffiths CJ, Martineau AR, Robinson S, Yu C, Poulton S, Kirkby JC, Stocks J, Hooper R, Shaheen SO, et al. Prenatal vitamin d supplementation and child respiratory health: a randomised controlled trial. PLoS One. 2013;8(6):e66627.

27. Chawes BL, Bonnelykke K, Stokholm J, Vissing NH, Bjarnadottir E, Schoos AM, Wolsk HM, Pedersen TM, Vinding RK, Thorsteinsdottir S, et al. Effect of vitamin D3 supplementation during pregnancy on risk of persistent wheeze in the offspring: a randomized clinical trial. JAMA. 2016;315(4):353-61.

28. Litonjua AA, Carey VJ, Laranjo N, Harshfield BJ, McElrath TF, O'Connor GT, Sandel M, Iverson RE Jr, Lee-Paritz A, Strunk RC, et al. Effect of prenatal supplementation with vitamin $D$ on asthma or recurrent wheezing in offspring by age 3 years: the VDAART randomized clinical trial. JAMA. 2016;315(4):362-70.

29. de Jongh RT, Crozier SR, D'Angelo S, Pike KC, Roberts G, Lucas JS, Inskip H, Godfrey KM, Cooper C, Harvey NC. Maternal 25-hydroxyvitamin D levels in relation to offspring respiratory symptoms and infections. Eur Respir J. 2014; 43(4):1181-3. 
30. Morales E, Romieu I, Guerra S, Ballester F, Rebagliato M, Vioque J, Tardon A, Rodriguez Delhi C, Arranz L, Torrent M, et al. Maternal vitamin D status in pregnancy and risk of lower respiratory tract infections, wheezing, and asthma in offspring. Epidemiology. 2012;23(1):64-71.

31. Zosky GR, Hart PH, Whitehouse AJ, Kusel MM, Ang W, Foong RE, Chen L, Holt PG, Sly PD, Hall GL. Vitamin D deficiency at 16 to 20 weeks' gestation is associated with impaired lung function and asthma at 6 years of age. Ann Am Thorac Soc. 2014;11(4):571-7.

32. Maslova E, Hansen S, Thorne-Lyman AL, Jensen CB, Strom M, Cohen A, Nielsen NO, Olsen SF. Predicted vitamin D status in mid-pregnancy and child allergic disease. Pediatr Allergy Immunol. 2014;25(7):706-13.

33. Wills AK, Shaheen SO, Granell R, Henderson AJ, Fraser WD, Lawlor DA. Maternal 25-hydroxyvitamin D and its association with childhood atopic outcomes and lung function. Clin Exp Allergy. 2013;43(10):1180-8.

34. Magnus MC, Stene LC, Nafstad P, Stigum H, London SJ, Nystad W. Prospective study of maternal mid-pregnancy 25-hydroxyvitamin D level and early childhood respiratory disorders. Paediatr Perinat Epidemiol. 2013; 27(6):532-41.

35. Chiu CY, Huang SY, Peng YC, Tsai MH, Hua MC, Yao TC, Yeh KW, Huang JL. Maternal vitamin D levels are inversely related to allergic sensitization and atopic diseases in early childhood. Pediatr Allergy Immunol. 2015;26(4):337-43.

36. Hansen S, Maslova E, Strom M, Linneberg A, Halldorsson TI, Granstrom C, Dahl R, Hoffmann HJ, Olsen SF. The long-term programming effect of maternal 25-hydroxyvitamin D in pregnancy on allergic airway disease and lung function in offspring after 20 to 25 years of follow-up. J Allergy Clin Immunol. 2015:136(1):169-76. e162

37. Gazibara T, den Dekker HT, de Jongste JC, McGrath JJ, Eyles DW, Burne TH, Reiss IK, Franco OH, Tiemeier $\mathrm{H}$, Jaddoe WW, et al. Associations of maternal and fetal 25-hydroxyvitamin D levels with childhood lung function and asthma: the generation R study. Clin Exp Allergy. 2016;46(2):337-46.

38. Baiz N, Dargent-Molina P, Wark JD, Souberbielle JC, Annesi-Maesano I. Cord serum 25-hydroxyvitamin D and risk of early childhood transient wheezing and atopic dermatitis. J Allergy Clin Immunol. 2014;133(1):147-53.

39. Camargo CA Jr, Thadhani R, Silvers KM, Pattemore PK, Espinola JA, Crane J, Dench C, Duignan M, Epton MJ, Fishwick D, et al. Cord-blood 25hydroxyvitamin $\mathrm{D}$ levels and risk of respiratory infection, wheezing, and asthma. Pediatrics. 2011;127(1):e180-7.

40. Stelmach I, Majak P, Jerzynska J, Podlecka D, Stelmach W, Polanska K, Gromadzinska J, Wasowicz W, Hanke W. Cord serum 25-hydroxyvitamin D correlates with early childhood viral-induced wheezing. Respir Med. 2015; 109(1):38-43.

41. Chawes BL, Bonnelykke K, Jensen PF, Schoos AM, Heickendorff L, Bisgaard H. Cord blood 25(OH)-vitamin D deficiency and childhood asthma, allergy and eczema: the COPSAC2000 birth cohort study. PLoS One. 2014;9(6):e99856.

42. Junge KM, Bauer T, Geissler S, Hirche F, Thurmann L, Bauer M, Trump S, Bieg $M$, Weichenhan D, Gu L, et al. Increased vitamin D levels at birth and in early infancy increase offspring allergy risk-evidence for involvement of epigenetic mechanisms. J Allergy Clin Immunol. 2016;137(2):610-3.

43. Palmer DJ, Sullivan TR, Skeaff CM, Smithers LG, Makrides M. Higher cord blood 25-hydroxyvitamin D concentrations reduce the risk of early childhood eczema: in children with a family history of allergic disease. World Allergy Organ J. 2015;8(1):28.

44. Visness CM, Sandel MT, O'Connor G, Gern JE, Jaffee KF, Wood RA, Kattan M, Bloomberg GR, Dresen A, Gergen PJ, et al. Cord blood vitamin D concentrations are unrelated to atopy and wheeze in 2 diverse birth cohort studies. J Allergy Clin Immunol. 2015;136(4):1108-10. e1102

45. Wolsk HM, Harshfield BJ, Laranjo N, Carey VJ, O'Connor G, Sandel M, Strunk RC, Bacharier LB, Zeiger RS, Schatz M, et al. Vitamin D supplementation in pregnancy, prenatal 25(OH)D levels, race, and subsequent asthma or recurrent wheeze in offspring: secondary analyses from the vitamin D antenatal asthma reduction trial. J Allergy Clin Immunol. 2017;140(5):1423-29.e5.

46. Hollams EM, Teo SM, Kusel M, Holt BJ, Holt KE, Inouye M, De Klerk NH, Zhang G, Sly PD, Hart PH, et al. Vitamin D over the first decade and susceptibility to childhood allergy and asthma. J Allergy Clin Immunol. 2017; 139(2):472-81. e479

47. Wegienka G, Havstad S, Zoratti EM, Kim H, Ownby DR, Johnson CC. Association between vitamin $\mathrm{D}$ levels and allergy-related outcomes vary by race and other factors. J Allergy Clin Immunol. 2015;136(5):1309-1314 e1301-1304.
48. Miyake Y, Sasaki S, Tanaka K, Hirota Y. Dairy food, calcium and vitamin D intake in pregnancy, and wheeze and eczema in infants. Eur Respir J. 2010; 35(6):1228-34

49. Devereux G, Litonjua AA, Turner SW, Craig LCA, McNeill G, Martindale S, Helms PJ, Seaton A, Weiss ST. Maternal vitamin D intake during pregnancy and early childhood wheezing. Am J Clin Nutr. 2007;85(3):853-9.

50. Allan KM, Prabhu N, Craig LC, McNeill G, Kirby B, McLay J, Helms PJ, Ayres JG, Seaton A, Turner SW, et al. Maternal vitamin D and E intakes during pregnancy are associated with asthma in children. Eur Respir J. 2015;45(4):1027-36.

51. Camargo CA Jr, Rifas-Shiman SL, Litonjua AA, Rich-Edwards JW, Weiss ST, Gold DR, Kleinman K, Gillman MW. Maternal intake of vitamin D during pregnancy and risk of recurrent wheeze in children at 3 y of age. Am J Clin Nutr. 2007:85(3):788-95.

52. Maslova E, Hansen S, Jensen CB, Thorne-Lyman AL, Strom M, Olsen SF. Vitamin D intake in mid-pregnancy and child allergic disease - a prospective study in 44,825 Danish mother-child pairs. BMC Pregnancy Childbirth. 2013;13(1):199.

53. Erkkola M, Kaila M, Nwaru BI, Kronberg-Kippila C, Ahonen S, Nevalainen J, Veijola R, Pekkanen J, Ilonen J, Simell O, et al. Maternal vitamin D intake during pregnancy is inversely associated with asthma and allergic rhinitis in 5-year-old children. Clin Exp Allergy. 2009;39(6):875-82.

54. Miyake $Y$, Tanaka K, Okubo H, Sasaki S, Arakawa M. Maternal consumption of dairy products, calcium, and vitamin D during pregnancy and infantile allergic disorders. Ann Allergy Asthma Immunol. 2014;113(1):82-7.

55. Anderson LN, Chen Y, Omand JA, Birken CS, Parkin PC, To T, Maguire JL. Vitamin D exposure during pregnancy, but not early childhood, is associated with risk of childhood wheezing. J Dev Orig Health Dis. 2015;6(4):308-16.

56. Back O, Blomquist HK, Hernell O, Stenberg B. Does vitamin D intake during infancy promote the development of atopic allergy? Acta Derm Venereol. 2009:89(1):28-32.

57. Hypponen E, Sovio U, Wjst M, Patel S, Pekkanen J, Hartikainen AL, Jarvelinb MR. Infant vitamin d supplementation and allergic conditions in adulthood: northern Finland birth cohort 1966. Ann N Y Acad Sci. 2004;1037:84-95.

58. von Mutius E, Martinez FD. Inconclusive results of randomized trials of prenatal vitamin $D$ for asthma prevention in offspring: curbing the enthusiasm. JAMA. 2016;315(4):347-8.

59. Litonjua AA, Weiss ST. Is vitamin D deficiency to blame for the asthma epidemic? J Allergy Clin Immunol. 2007;120(5):1031-5.

60. Wjst M. Is vitamin D supplementation responsible for the allergy pandemic? Curr Opin Allergy Clin Immunol. 2012;12(3):257-62.

61. Autier P, Boniol M, Pizot C, Mullie P. Vitamin D status and ill health: a systematic review. Lancet Diabetes Endocrinol. 2014;2(1):76-89.

62. Burke $H$, Leonardi-Bee J, Hashim A, Pine-Abata H, Chen Y, Cook DG, Britton JR, McKeever TM. Prenatal and passive smoke exposure and incidence of asthma and wheeze: systematic review and meta-analysis. Pediatrics. 2012; 129(4):735-44.

63. King ME, Mannino DM, Holguin F. Risk factors for asthma incidence. A review of recent prospective evidence. Panminerva Med. 2004:46(2):97-110.

64. Kasahara AK, Singh RJ, Noymer A. Vitamin D (25OHD) serum seasonality in the United States. PLoS One. 2013;8(6):e65785.

65. Jones KS, Assar S, Vanderschueren D, Bouillon R, Prentice A, Schoenmakers I. Predictors of $25(\mathrm{OH}) \mathrm{D}$ half-life and plasma $25(\mathrm{OH}) \mathrm{D}$ concentration in the Gambia and the UK. Osteoporos Int. 2015;26(3):1137-46.

66. Pekkanen J, Pearce N. Defining asthma in epidemiological studies. Eur Respir J. 1999:14(4):951-7.

67. Canova C, Harris JM, Mills P, White C, Moffat S, Shread L, Cullinan P. Epidemiological measures of childhood asthma: cross-sectional and longitudinal consistency. Respir Med. 2012;106(9):1226-35.

68. Depner M, Fuchs O, Genuneit J, Karvonen AM, Hyvarinen A, Kaulek V, Roduit C, Weber J, Schaub B, Lauener R, et al. Clinical and epidemiologic phenotypes of childhood asthma. Am J Respir Crit Care Med. 2014;189(2):129-38.

69. Cornish RP, Henderson J, Boyd AW, Granell R, Van Staa T, Macleod J. Validating childhood asthma in an epidemiological study using linked electronic patient records. BMJ Open. 2014:4(4):e005345.

70. Cassim R, Russell MA, Lodge CJ, Lowe AJ, Koplin JJ, Dharmage SC. The role of circulating 25 hydroxyvitamin $D$ in asthma: a systematic review. Allergy. 2015;70(4):339-54 